Claremont Colleges

Scholarship@ Claremont

All HMC Faculty Publications and Research

HMC Faculty Scholarship

$1-1-2017$

\title{
First Passage Statistics for the Capture of a Brownian Particle by a Structured Spherical Target with Multiple Surface Traps
}

\author{
Alan E. Lindsay \\ University of Notre Dame \\ Andrew Bernoff \\ Harvey Mudd College \\ Michael J. Ward \\ University of British Columbia
}

\section{Recommended Citation}

Lindsay, A. E., Bernoff, A. J., \& Ward, M. J. (2017). First Passage Statistics for the Capture of a Brownian Particle by a Structured Spherical Target with Multiple Surface Traps. Multiscale Model. Simul., 15(1), 74-109. http://epubs.siam.org/doi/abs/10.1137/ 16M1077659 


\title{
FIRST PASSAGE STATISTICS FOR THE CAPTURE OF A BROWNIAN PARTICLE BY A STRUCTURED SPHERICAL TARGET WITH MULTIPLE SURFACE TRAPS*
}

\author{
ALAN E. LINDSAY ${ }^{\dagger}$, ANDREW J. BERNOFF ${ }^{\ddagger}$, AND MICHAEL J. WARD ${ }^{\S}$
}

\begin{abstract}
We study the first passage time problem for a diffusing molecule in an enclosed region to hit a small spherical target whose surface contains many small absorbing traps. This study is motivated by two examples of cellular transport. The first is the intracellular process through which proteins transit from the cytosol to the interior of the nucleus through nuclear pore complexes that are distributed on the nuclear surface. The second is the problem of chemoreception, in which cells sense their surroundings through diffusive contact with receptors distributed on the cell exterior. Using a matched asymptotic analysis in terms of small absorbing pore radius, we derive and numerically verify a high order expansion for the capacitance of the structured target which incorporates surface effects and gives explicit information on interpore interaction through a Coulomb-type discrete energy with additional logarithmic dependencies. In the large $N$ dilute surface trap fraction limit, a single homogenized Robin boundary condition $\partial_{n} v+\kappa v=0$ is derived in which $\kappa$ depends on the total absorbing fraction, the characteristic pore scale, and parameters relating to interpore interactions.
\end{abstract}

Key words. Brownian motion, Berg-Purcell, singular perturbations, homogenization, mean first passage time

AMS subject classifications. 35B25, 35C20, 35J05, 35J08

DOI. $10.1137 / 16 \mathrm{M} 1077659$

1. Introduction. Many biological systems utilize diffusive transport to enable the delivery of reactants or signaling molecules to specific spatial locations where they can initiate some biological function. Depending on the particular setting, the spatial region may have a complex geometry that contains obstacles to the diffusive motion together with a heterogeneous distribution of delivery sites. The effectiveness of the diffusion mechanism in light of these factors can be understood by studying the first passage time statistics of Brownian walkers to small stationary targets. In many biological settings, the number of individual molecules is typically very large, and so the mean first passage time (MFPT) is an important quantity and the focus of many recent studies $[47,24,9,43,1,32,38,37,46,15,10,12,16]$.

One key intracellular process is the transport of proteins between the cell cytoplasm and the interior of the nucleus (cf. [28, 29, 22]). The cell nucleus is typically spherical or ellipsoidal in shape and occupies roughly $10 \%$ of the total cell volume. The nuclear surface is an impermeable double membrane that separates the chromosomes from the cell cytoplasm [50]. The transit of proteins to the interior of the nucleus is crucial to the development process and is regulated by the distribution of nuclear pore complexes (NPCs) that cover the nuclear surface [50]. The nuclear radius is roughly

\footnotetext{
*Received by the editors May 31, 2016; accepted for publication (in revised form) November 10, 2016; published electronically January 5, 2017.

http://www.siam.org/journals/mms/15-1/M107765.html

Funding: The first author was supported by NSF grant DMS-1516753. The second author was supported by Simons Foundation grant 317319. The third author was supported by NSERC (Canada) Discovery grant 81541.

${ }^{\dagger}$ Department of Applied and Computational Math \& Statistics, Notre Dame University, South Bend, IN 46656 (a.lindsay@nd.edu).

${ }^{\ddagger}$ Department of Mathematics, Harvey Mudd College, Claremont, CA 91711 (ajb@hmc.edu).

$\S^{\S}$ pepartment of Mathematics, University of British Columbia, Vancouver, BC, Canada (ward@ math.ubc.ca).
} 
4 microns, and there are estimated to be approximately $N=2000$ nanotraps, each of estimated radius 25 nanometers (cf. [29, 41]). This implies that roughly $2 \%$ of the boundary of the nucleus is covered by nanotraps. One aim of this study is to analyze a simple model for how the number and distribution of NPCs regulate the timescale of transport over the nuclear barrier.

A second biophysical process motivating this study is chemoreception. Cells sense their surroundings through contact with diffusing molecules at receptor sites distributed over their exterior membrane. Even if a small surface area fraction of the cell exterior is occupied by receptors, its sensing ability can be near optimal, provided the receptors are numerous and well distributed over the cell surface [4]. In this scenario, our aim is to give a detailed first principles description of how the number and spatial configuration of absorbing receptors dictate the statistics of contact events.

Motivated by these biological scenarios, we will analyze the MFPT and the standard deviation for Brownian motion in a bounded three-dimensional (3-D) domain $\Omega \subset \mathbb{R}^{3}$ when there is a small, but structured, target sphere $\Omega_{\varepsilon}$ of radius $\varepsilon \ll 1$ centered at some $\mathbf{x}_{0} \in \Omega$. For simplicity, we will neglect the often important effect of directed transport to the target site (cf. [28, 29]) and instead assume that there is a simple Brownian motion to the target. The complicating factor in this problem is that the boundary $\partial \Omega_{\varepsilon}$ of the target sphere is highly heterogeneous, of mixed DirichletNeumann type, and is assumed to consist of many small locally circular absorbing patches, or nanotraps, on an otherwise reflecting surface. For a Brownian particle with diffusivity $D$ starting at $\mathbf{x} \in \Omega \backslash \Omega_{\varepsilon}$, the MFPT $w(\mathbf{x})$ for the Brownian particle to first encounter any of the absorbing surface patches is well known to satisfy the narrow capture problem (cf. [40, 23])

$$
\begin{gathered}
\Delta w=-\frac{1}{D}, \quad \mathbf{x} \in \Omega \backslash \Omega_{\varepsilon} ; \quad \partial_{n} w=0, \quad \mathbf{x} \in \partial \Omega, \\
w=0, \quad \mathbf{x} \in \partial \Omega_{\varepsilon a} ; \quad \partial_{n} w=0, \quad \mathbf{x} \in \partial \Omega_{\varepsilon r},
\end{gathered}
$$

where $\partial \Omega_{\varepsilon a}$ is the multiply-connected absorbing set and $\partial \Omega_{\varepsilon r}$ is the reflecting portion of the surface of the target. Here $\Omega_{\varepsilon}=\mathbf{x}_{0}+\varepsilon \Omega_{0}$, where $\Omega_{0}$ is the unit sphere centered at the origin $\mathbf{y}=0$, where $\mathbf{y} \equiv \varepsilon^{-1}\left(\mathbf{x}-\mathbf{x}_{0}\right)$. We write $\partial \Omega_{0}=\Gamma_{a} \cup \Gamma_{r}$, where $\Gamma_{a}$ and $\Gamma_{r}$ are the absorbing and reflecting portions of this reference unit sphere (see Figure 1 for the geometry).

In the limit $\varepsilon \rightarrow 0$, a two-term expansion for the MFPT $w(\mathbf{x})$ (cf. [11]; see

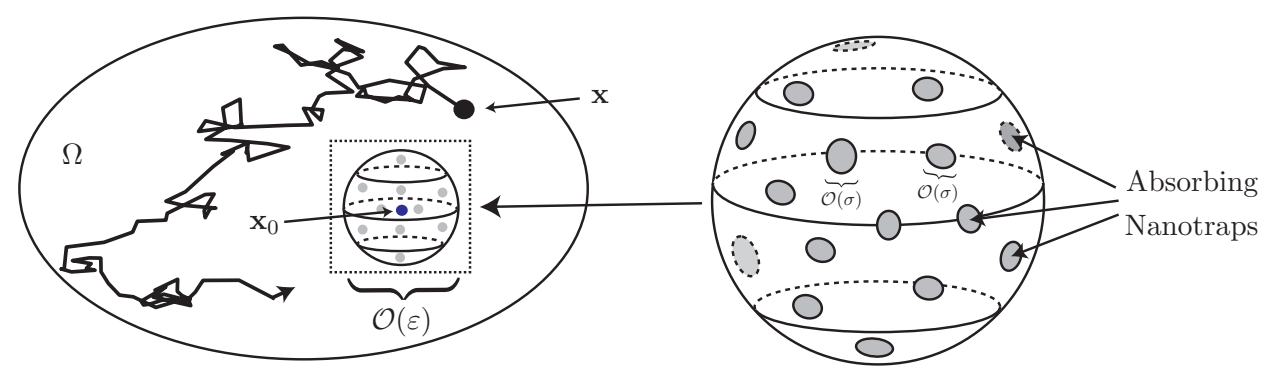

FIG. 1. Schematic of a structured target centered at $\mathbf{x}_{0} \in \Omega$ of radius $\mathcal{O}(\varepsilon)$ that has a collection of $N$ absorbing surface patches, referred to here as nanotraps, of common radius $\mathcal{O}(\sigma)$. A random walker starts at location $\mathbf{x} \in \Omega$ and eventually reaches an absorbing nanotrap. 
Principal Result 2.1 in section 2) is

$$
w(\mathbf{x})=\frac{|\Omega|}{4 \pi C_{0} D \varepsilon}\left[1-4 \pi C_{0} \varepsilon\left(G_{m}\left(\mathbf{x} ; \mathbf{x}_{0}\right)-R_{m}\left(\mathbf{x}_{0}\right)\right)+\mathcal{O}\left(\varepsilon^{2}\right)\right],
$$

where $G_{m}\left(\mathbf{x} ; \mathbf{x}_{0}\right)$ is the Neumann Green's function of $\Omega$ with regular part $R_{m}\left(\mathbf{x}_{0}\right)$ satisfying

$$
\Delta G_{m}=\frac{1}{|\Omega|}-\delta(\mathbf{x}-\boldsymbol{\xi}), \quad \mathbf{x} \in \Omega ; \quad \partial_{n} G_{m}=0, \quad \mathbf{x} \in \partial \Omega
$$

$G_{m}\left(\mathbf{x} ; \mathbf{x}_{0}\right) \sim \frac{1}{4 \pi\left|\mathbf{x}-\mathbf{x}_{0}\right|}+R_{m}\left(\mathbf{x}_{0}\right)+o(1) \quad$ as $\quad \mathbf{x} \rightarrow \mathbf{x}_{0} ; \quad \int_{\Omega} G_{m}\left(\mathbf{x} ; \mathbf{x}_{0}\right) d \mathbf{x}=0$.

The average MFPT, based on assuming a uniform distribution of starting locations, is calculated from (1.2) as

$$
\tau \equiv \frac{1}{\left|\Omega \backslash \Omega_{\varepsilon}\right|} \int_{\Omega \backslash \Omega_{\varepsilon}} w d \mathbf{x}=\frac{|\Omega|}{4 \pi C_{0} D \varepsilon}\left[1+4 \pi \varepsilon C_{0} R_{m}\left(\mathbf{x}_{0}\right)+\mathcal{O}\left(\varepsilon^{2}\right)\right] .
$$

The quantity $\tau$ provides the broadest measure of the timescale for the capture of a Brownian particle by the structured target sphere. The term $R_{m}\left(\mathbf{x}_{0}\right)$ captures the dependence of $\tau$ on the spatial location of the target sphere in $\Omega$, while the spatially uniform parameter $C_{0}$, known as the capacitance and defined below by the local problem (1.5), encodes information on the number and spatial distribution of absorbing nanotraps on the surface of the target.

As an extension to the MFPT analysis, in section 2 we follow [16] to calculate an asymptotic expansion for the standard deviation $\Sigma$ of the first passage process. This new result, summarized in Principal Result 2.2 below, shows that in the region where $\left|\mathbf{x}-\mathbf{x}_{0}\right| \gg \mathcal{O}(\varepsilon)$, the first two terms in the asymptotic expansion of $\Sigma$ are independent of the starting point for the Brownian motion and agree precisely with the two-term asymptotics for the average MFPT $\tau$ of (1.4). This result shows that for a pure Brownian walk there is a significant spread in the distribution of first capture times about the mean.

However, the main focus of this paper is to determine the capacitance $C_{0}$ when the spherical trap has a heterogeneous distribution of absorbing sites, such as displayed in Figure 1. The capacitance $C_{0}$ is defined in terms of a canonical exterior potential-field problem with a prescribed flux at infinity, formulated as

$$
\begin{gathered}
\Delta v=0, \quad \mathbf{y} \in \mathbb{R}^{3} \backslash \Omega_{0} ; \quad v=0, \quad \mathbf{y} \in \Gamma_{a}, \quad \partial_{n} v=0, \quad \mathbf{y} \in \Gamma_{r}, \\
\lim _{R \rightarrow \infty} \int_{\partial \Omega_{R}} \partial_{n} v d s=-4 \pi,
\end{gathered}
$$

where $\partial \Omega_{R} \equiv\left\{\mathbf{y} \in \mathbb{R}^{3}|| \mathbf{y} \mid=R\right\}$ and $\Omega_{R}$ contains $\Omega_{0}$. In terms of this solution, $C_{0}$ is defined by the far-field behavior

$$
v=\frac{1}{|\mathbf{y}|}-\frac{1}{C_{0}}\left[1-\frac{\mathbf{p} \cdot \mathbf{y}}{|\mathbf{y}|^{3}}\right]+\cdots \quad \text { as } \quad|\mathbf{y}| \rightarrow \infty,
$$

where $\mathbf{p}$ is the dipole moment associated with the target. We will consider the case where $\Omega_{0}$ is the unit sphere that has $N$ small nonoverlapping absorbing locally circular 
surface nanotraps $\Gamma_{a}=\cup_{k=1}^{N} \partial \Omega_{k}$ with locations $\mathbf{y}_{k}$ and radii $\sigma a_{k}$ for $k=1, \ldots, N$, with $\left|\mathbf{y}_{j}-\mathbf{y}_{k}\right| \gg \mathcal{O}(\sigma)$ for $j \neq k$, where $\sigma \ll 1$. In spherical coordinates, we write

$$
\begin{aligned}
\mathbf{y}_{k} & =\left(\sin \theta_{k} \cos \phi_{k}, \sin \theta_{k} \sin \phi_{k}, \cos \theta_{k}\right)^{T}, \\
\partial \Omega_{k} & \equiv\left\{(\theta, \phi) \mid\left(\theta-\theta_{k}\right)^{2}+\sin ^{2}\left(\theta_{k}\right)\left(\phi-\phi_{k}\right)^{2} \leq \sigma^{2} a_{k}^{2}\right\},
\end{aligned}
$$

so that the $k$ th nanotrap area is $\left|\partial \Omega_{k}\right| \approx \pi a_{k}^{2} \sigma^{2}$. In other cases where the target is perfectly absorbing, explicit formulae for $C_{0}$ are well known for some regular geometries such as spheres, hemispheres, and ellipsoids [11]. In the simpler 2-D context, the case of small circular targets with a nonuniform partially absorbing boundary was analyzed in [32].

For our 3-D structured target problem (1.5), in section 3 we will use the method of matched asymptotic expansions in the limit $\sigma \rightarrow 0$ and for finite $N$ to determine an explicit four-term asymptotic expansion for $C_{0}$, which depends on the radii $a_{k}$ and locations $\mathbf{y}_{k}$ of the centers of the nanotraps for $k=1, \ldots, N$. The analysis to determine $C_{0}$ is related to that done for the narrow escape problem in [12] in that it relies on detailed knowledge of a certain surface Neumann Green's function together with the introduction of certain logarithmic switchback terms (cf. [30]) that arise from the subdominant logarithmic singularity of this Green's function. This surface Green's function $G\left(\mathbf{y}, \mathbf{y}_{0}\right)$ is the solution to

$$
\begin{aligned}
& \Delta G=0, \quad r=|\mathbf{y}|>1, \quad 0<\theta<\pi, \quad 0<\phi<2 \pi ; \quad G \sim \frac{1}{4 \pi|\mathbf{y}|} \quad \text { as } \quad|\mathbf{y}| \rightarrow \infty, \\
& (1.7 \mathrm{~b}) \quad-\left.\partial_{r} G\right|_{r=1}=\frac{1}{\sin \theta_{0}} \delta\left(\phi-\phi_{0}\right) \delta\left(\theta-\theta_{0}\right), \quad 0<\theta<\pi, \quad 0<\phi<2 \pi,
\end{aligned}
$$

where $\mathbf{y}_{0}=\left(\sin \theta_{0} \cos \phi_{0}, \sin \theta_{0} \sin \phi_{0}, \cos \theta_{0}\right)^{T}$, which is given explicitly by (cf. [35], $[25])$

$$
G\left(\mathbf{y} ; \mathbf{y}_{0}\right)=\frac{1}{2 \pi}\left[\frac{1}{\left|\mathbf{y}-\mathbf{y}_{0}\right|}-\frac{1}{2} \log \left(\frac{1-\mathbf{y} \cdot \mathbf{y}_{0}+\left|\mathbf{y}-\mathbf{y}_{0}\right|}{|\mathbf{y}|-\mathbf{y} \cdot \mathbf{y}_{0}}\right)\right] .
$$

Our main result for $C_{0}$ is given below in Principal Result 3.1 of section 3. For the special case where the nanotraps have a common radius $\sigma \ll 1$, but with an arbitrary nonoverlapping spatial distribution on the surface, we will show that $C_{0}=C_{0}\left(\mathbf{y}_{1}, \ldots, \mathbf{y}_{N}\right)$ satisfies

(1.8a)

$$
\frac{1}{C_{0}}=\frac{\pi}{N \sigma}\left[1+\frac{\sigma}{\pi}\left(\log \left(2 e^{-3 / 2} \sigma\right)+\frac{4}{N} \mathcal{H}\left(\mathbf{y}_{1}, \ldots, \mathbf{y}_{N}\right)\right)+\mathcal{O}\left(\sigma^{2} \log \left(\frac{\sigma}{2}\right)\right)\right],
$$

where the discrete energy $\mathcal{H}\left(\mathbf{y}_{1}, \ldots, \mathbf{y}_{N}\right)$, representing both Coulombic and logarithmic internanotrap interactions, is

$$
\begin{aligned}
\mathcal{H}\left(\mathbf{y}_{1}, \ldots, \mathbf{y}_{N}\right) \equiv 2 \pi \sum_{j=1}^{N} \sum_{k=j+1}^{N} G\left(\mathbf{y}_{j} ; \mathbf{y}_{k}\right) & =\sum_{j=1}^{N} \sum_{k=j+1}^{N} g\left(\left|\mathbf{y}_{j}-\mathbf{y}_{k}\right|\right) \\
& \text { where } g(\mu) \equiv \frac{1}{\mu}+\frac{1}{2} \log \left(\frac{\mu}{2+\mu}\right) .
\end{aligned}
$$

Since on the interval $0<\mu<2, g(\mu)$ is a monotone decreasing, positive, and convex function, the spatial configuration $\left\{\mathbf{y}_{1}, \ldots, \mathbf{y}_{N}\right\}$ of nanotraps that minimizes $\mathcal{H}$, and 
consequently maximizes $C_{0}$, will be (roughly) uniformly distributed over the surface of the target sphere. This discrete optimization problem for points on the sphere is a generalization of the classical Fekete point problems of [20, 5, 39, 27, 44, 7]. In addition, as a result of the different Green's functions involved, this problem is different from the discrete optimization problem derived in [12] to minimize the average MFPT for the narrow escape problem. For finite $N$, in section 4.1 we examine numerically the effect of both the spatial distribution of the nanotraps and the fragmentation of the nanotrap set. For the case of a single nanotrap, where $\mathcal{H} \equiv 0$, in section 6 an exact solution to (1.5) in terms of a dual Legendre series, inspired by [47, 14], is derived to provide partial verification of $(1.8 \mathrm{a})$.

The canonical dimensionless problem (1.5) is equivalent to the well-known biophysical problem of analyzing how diffusing ligands bind to cell surface receptors on a spherical surface of radius $R_{0}$ (cf. $[4,31]$ ). In this context, ligands are modeled as point Brownian particles with diffusivity $D_{0}$ that are trapped upon first contact with any disk-shaped surface receptor of radius $a_{0}$. The corresponding steady-state dimensional ligand concentration $u(\mathbf{x})$ satisfies

$$
\begin{gathered}
\Delta u=0, \quad|\mathbf{x}| \geq R_{0} ; \quad u \sim 1-C /|\mathbf{x}| \quad \text { as } \quad|\mathbf{x}| \rightarrow \infty, \\
u=0, \quad \mathbf{x} \in \partial \Omega_{a}, \quad \partial_{n} u=0, \quad \mathbf{x} \in \partial \Omega_{r},
\end{gathered}
$$

where the absorbing set $\partial \Omega_{a}$ on the sphere consists of $N$ locally circular cell surface receptor patches of a common radius $a_{0} \ll 1$, while $\partial \Omega_{r}$ is the nonbinding (reflecting) part of the surface of the sphere. In terms of the capacitance $C$, as defined by the far-field behavior (1.9a), the flux $J$ of ligands to the surface receptors is $J \equiv$ $\left.\int_{\partial \Omega_{a}} D_{0} u_{r}\right|_{r=R_{0}} d s=4 \pi D_{0} C$. When the entire surface is absorbing, then $u=1-$ $R_{0} /|\mathbf{x}|$, so that $C=R_{0}$, which yields Smoluchowski's classical result $J_{\mathrm{sm}}=4 \pi D_{0} R_{0}$. When there is only partial coverage by identical disk-shaped receptors on the sphere, the physically motivated flux-based analysis of Berg and Purcell [4] postulated, for a spatially uniform arrangement of such receptors, that

$$
J_{\mathrm{bp}}=4 \pi D_{0} C_{\mathrm{bp}}, \quad C_{\mathrm{bp}}=R_{0} \frac{N a_{0}}{N a_{0}+\pi R_{0}},
$$

provided that the receptor surface area coverage $f \equiv \frac{N}{4}\left(a_{0} / R_{0}\right)^{2}$ is small. This effective capacitance $C_{\mathrm{bp}}$ can then be used to homogenize the highly nonuniform boundary conditions $(1.9 \mathrm{~b})$ by a uniform Robin condition $D_{0} \partial_{n} u+\kappa u=0$ on the entire surface $r=R_{0}$. By solving (1.9a) with this Robin condition, we readily identify that $\kappa=D_{0} C /\left(R_{0}\left(R_{0}-C\right)\right)$, and so using the Berg-Purcell effective capacitance (1.10a) and eliminating $N$ in terms of $f$, the leakage $\kappa_{\text {bp }}$ is identified as

$$
\kappa_{\mathrm{bp}}=\left(\frac{D_{0}}{R_{0}}\right)\left(\frac{4 f}{\pi \sigma}\right), \quad \sigma \equiv \frac{a_{0}}{R_{0}} \ll 1, \quad f \equiv \frac{N \sigma^{2}}{4} .
$$

More detailed studies [45, 51, 2, 3, 34] and fittings of the leakage parameter $\kappa$ in a boundary homogenization procedure for nanotraps on either the sphere or on a flat plane are discussed in section 4. By taking the limit $N \gg 1$ in our expression for the capacitance $C_{0}$ for a uniformly distributed configuration of nanotraps of a common radius $\sigma$, our result in section 4 for the low surface nanotrap coverage limit $f=\mathcal{O}\left(-\sigma^{2} \log \sigma\right) \ll 1$, and when cast in dimensional form, predicts that

$\kappa \sim\left(\frac{D_{0}}{R_{0}}\right)\left(\frac{4 f}{\pi \sigma}\right)\left[1-\frac{8 d_{1}}{\pi} \sqrt{f}+\frac{\sigma}{\pi} \log (\beta \sqrt{f})+\frac{2 d_{3} \sigma^{2}}{\pi \sqrt{f}}\right]^{-1}$, where $\beta \equiv 4 e^{-3 / 2} e^{4 d_{2}}$, 
and $\sigma \equiv\left(a_{0} / R_{0}\right) \ll 1$. Here $d_{j}$ for $j=1, \ldots, 3$ are coefficients in the large $N$ expansion of the energy $\mathcal{H}$ in $(1.8 \mathrm{~b})$ for a uniform distribution of nanotraps on the sphere of the form $\mathcal{H}=\frac{N^{2}}{4}-d_{1} N^{3 / 2}+\frac{1}{8} N \log N+d_{2} N+d_{3} N^{1 / 2}+\mathcal{O}(\log N)$. The leading term in (1.11) agrees with the Berg-Purcell result (1.10b), while the $8 d_{1} \sqrt{f} / \pi$ term theoretically explains one of the parameter fittings in $[3,34]$. A further discussion of this result and a comparison to other results in the literature, notably those in $[2,3,34,51]$, are discussed in section 4 . Finally, in section 7 we briefly summarize our main results and discuss a few open problems worthy of further study.

2. Asymptotic analysis of the MFPT and the variance. In this section we calculate the standard deviation of the first passage time and compare it with the asymptotic result for the MFPT, as given in Principal Result 3.1 of [11]. The two-term expansion for the MFPT in terms of the capacitance $C_{0}$ of the target, as defined by (1.5), is as follows.

Principal Result 2.1 (from [11]). In the limit $\varepsilon \rightarrow 0$ of small trap radius, the MFPT $w$, satisfying (1.1), is given asymptotically in the outer region $\left|\mathbf{x}-\mathbf{x}_{0}\right| \gg \mathcal{O}(\varepsilon)$ by

$$
w \sim \frac{|\Omega|}{4 \pi C_{0} D \varepsilon}\left[1-4 \pi \varepsilon C_{0} G_{m}\left(\mathbf{x} ; \mathbf{x}_{0}\right)+4 \pi \varepsilon C_{0} R_{m}\left(\mathbf{x}_{0}\right)+\mathcal{O}\left(\varepsilon^{2}\right)\right],
$$

where the Neumann Green's function $G_{m}$ and its regular part $R_{m}$ satisfy (1.3). The average MFPT $\tau \sim|\Omega|^{-1} \int_{\Omega} w d \mathbf{x}$, based on assuming a uniform distribution of starting points $\mathbf{x}$ for the Brownian walk, is given in (1.4).

Next, we use the method of matched asymptotic expansions to calculate the second moment $\mathbb{T}$, which satisfies (cf. [16])

$$
\begin{array}{r}
\Delta \mathbb{T}=-\frac{2}{D} w, \quad \mathbf{x} \in \Omega \backslash \Omega_{\varepsilon} ; \quad \partial_{n} \mathbb{T}=0, \quad \mathbf{x} \in \partial \Omega, \\
\mathbb{T}=0, \quad \mathbf{x} \in \partial \Omega_{\varepsilon a}, \quad \partial_{n} \mathbb{T}=0, \quad \mathbf{x} \in \partial \Omega_{\varepsilon_{r}} .
\end{array}
$$

Since $w=\varepsilon^{-1} w_{0}+w_{1}+\cdots$ in the outer region, as given in Principal Result 2.1, we expand $\mathbb{T}$ there as

$$
\mathbb{T}=\varepsilon^{-2} \mathbb{T}_{0}+\varepsilon^{-1} \mathbb{T}_{1}+\mathbb{T}_{2}+\cdots,
$$

where $\mathbb{T}_{0}$ is an unknown constant. From (2.2a), we obtain that $\mathbb{T}_{k}$ for $k=1,2$ satisfies

$$
\Delta \mathbb{T}_{k}=-\frac{2}{D} w_{k-1}, \quad \mathbf{x} \in \Omega \backslash\left\{\mathbf{x}_{0}\right\} ; \quad \partial_{n} \mathbb{T}_{k}=0, \quad \mathbf{x} \in \partial \Omega,
$$

subject to a singularity condition as $\mathbf{x} \rightarrow \mathbf{x}_{0}$. Here $w_{k}$ for $k=0,1$ can be identified from (2.1). In the inner region near the sphere, we expand the inner solution $\mathcal{T}(\mathbf{y}) \equiv$ $\mathbb{T}\left(\mathbf{x}_{0}+\varepsilon \mathbf{y}\right)$, with $\mathbf{y} \equiv \varepsilon^{-1}\left(\mathbf{x}-\mathbf{x}_{0}\right)$, as

$$
\mathcal{T}=\varepsilon^{-2} \mathcal{T}_{0}+\varepsilon^{-1} \mathcal{T}_{1}+\mathcal{T}_{2}+\cdots
$$

Upon substituting (2.5) into (2.2b) and the PDE of (2.2a), we obtain that $\mathcal{T}_{k}$ for $k=0,1$ satisfies

$$
\Delta_{y} \mathcal{T}_{k}=0, \quad \mathbf{y} \notin \Omega_{0} ; \quad \mathcal{T}_{k}=0, \quad \mathbf{y} \in \Gamma_{a} ; \quad \partial_{n} \mathcal{T}_{k}=0, \quad \mathbf{y} \in \Gamma_{r} .
$$


The matching condition between the inner and outer solutions is that for $\mathbf{x} \rightarrow \mathbf{x}_{0}$ and $|\mathbf{y}| \rightarrow \infty$, we have

$$
\varepsilon^{-2} \mathbb{T}_{0}+\varepsilon^{-1} \mathbb{T}_{1}+\mathbb{T}_{2}+\cdots \sim \varepsilon^{-2} \mathcal{T}_{0}+\varepsilon^{-1} \mathcal{T}_{1}+\mathcal{T}_{2}+\cdots
$$

The leading order matching condition is that $\mathcal{T}_{0} \rightarrow \mathbb{T}_{0}$ as $|\mathbf{y}| \rightarrow \infty$. From (2.6), and by comparing with our canonical capacitance problem (1.5) for $v(\mathbf{y})$ and $C_{0}$, we conclude that

$$
\mathcal{T}_{0}=-C_{0} \mathbb{T}_{0} v
$$

Upon using the far-field behavior of $v$ from (1.5c), we obtain in terms of outer variables that

$$
\mathcal{T}_{0} \sim \mathbb{T}_{0}\left(1-\frac{\varepsilon C_{0}}{\left|\mathbf{x}-\mathbf{x}_{0}\right|}-\frac{\varepsilon^{2} \mathbf{p} \cdot\left(\mathbf{x}-\mathbf{x}_{0}\right)}{\left|\mathbf{x}-\mathbf{x}_{0}\right|^{3}}+\cdots\right) \quad \text { as } \quad|\mathbf{y}| \rightarrow \infty
$$

Then from (2.7) we obtain $\mathbb{T}_{1} \sim-C_{0} \mathbb{T}_{0} /\left|\mathbf{x}-\mathbf{x}_{0}\right|$ as $\mathbf{x} \rightarrow \mathbf{x}_{0}$. This local singularity behavior is equivalent to a Dirac source term $4 \pi C_{0} \mathbb{T}_{0} \delta\left(\mathbf{x}-\mathbf{x}_{0}\right)$ in the equation for $\mathbb{T}_{1}$. Therefore (2.4) for $k=1$ yields that

$$
\Delta \mathbb{T}_{1}=-\frac{2}{D} w_{0}+4 \pi C_{0} \mathbb{T}_{0} \delta\left(\mathbf{x}-\mathbf{x}_{0}\right), \quad \mathbf{x} \in \Omega ; \quad \partial_{n} \mathbb{T}_{1}=0, \quad \mathbf{x} \in \partial \Omega
$$

Upon applying the divergence theorem to (2.10) and using $w_{0}=|\Omega| /\left(4 \pi C_{0} D\right)$ from (2.1), we calculate $\mathbb{T}_{0}$ as

$$
\mathbb{T}_{0}=\frac{2 w_{0}|\Omega|}{4 \pi C_{0} D}=\frac{|\Omega|^{2}}{8 \pi^{2}\left(C_{0} D\right)^{2}} .
$$

The solution to (2.10) is written in terms of the Neumann Green's function $G_{m}$ of (1.3) as

$$
\mathbb{T}_{1}(\mathbf{x})=-4 \pi C_{0} \mathbb{T}_{0} G_{m}\left(\mathbf{x} ; \mathbf{x}_{0}\right)+\overline{\mathbb{T}}_{1},
$$

where $\overline{\mathbb{T}}_{1} \equiv|\Omega|^{-1} \int_{\Omega} \mathbb{T}_{1} d \mathbf{x}$ is a constant to be found. We then expand $\mathbb{T}_{1}$ as $\mathbf{x} \rightarrow \mathbf{x}_{0}$ to obtain

$$
\mathbb{T}_{1} \sim-\frac{C_{0} \mathbb{T}_{0}}{\left|\mathbf{x}-\mathbf{x}_{0}\right|}+\left[\overline{\mathbb{T}}_{1}-4 \pi C_{0} \mathbb{T}_{0} R_{m}\left(\mathbf{x}_{0}\right)\right]+o(1) \quad \text { as } \quad \mathbf{x} \rightarrow \mathbf{x}_{0} .
$$

Upon matching to the inner solution using (2.7) it follows that $\mathcal{T}_{1}$ satisfies (2.6) subject to the far-field behavior $\mathcal{T}_{1} \sim\left[\overline{\mathbb{T}}_{1}-4 \pi C_{0} \mathbb{T}_{0} R_{m}\left(\mathbf{x}_{0}\right)\right]$ as $|\mathbf{y}| \rightarrow \infty$, which has the solution

$$
\mathcal{T}_{1}=\left[\overline{\mathbb{T}}_{1}-4 \pi C_{0} \mathbb{T}_{0} R_{m}\left(\mathbf{x}_{0}\right)\right]\left(-C_{0} v\right) .
$$

By using the far-field behavior (1.5c) for $v$, the monopole term in $\mathcal{T}_{1}$ together with the dipole term in $\mathcal{T}_{0}$ in (2.9) leads to the singularity behavior for $\mathbb{T}_{2}$ that

$$
\mathbb{T}_{2} \sim-\frac{C_{0}\left[\overline{\mathbb{T}}_{1}-4 \pi C_{0} \mathbb{T}_{0} R_{m}\left(\mathbf{x}_{0}\right)\right]}{\left|\mathbf{x}-\mathbf{x}_{0}\right|}-\mathbb{T}_{0} \frac{\mathbf{p} \cdot\left(\mathbf{x}-\mathbf{x}_{0}\right)}{\left|\mathbf{x}-\mathbf{x}_{0}\right|^{3}} \quad \text { as } \quad \mathbf{x} \rightarrow \mathbf{x}_{0}
$$


From this singularity behavior, and together with (2.4) with $k=2$, we get

$$
\begin{aligned}
\Delta \mathbb{T}_{2}=-\frac{2}{D} w_{1}+4 \pi C_{0}\left[\overline{\mathbb{T}}_{1}-4 \pi C_{0} \mathbb{T}_{0} R_{m}\left(\mathbf{x}_{0}\right)\right] \delta\left(\mathbf{x}-\mathbf{x}_{0}\right)-\mathbb{T}_{0} \mathbf{p} \cdot \nabla_{\mathbf{x}} \delta\left(\mathbf{x}-\mathbf{x}_{0}\right), \quad \mathbf{x} \in \Omega ; \\
\quad \partial_{n} T_{2}=0, \quad \mathbf{x} \in \partial \Omega .
\end{aligned}
$$

By applying the divergence theorem on (2.16) and using $\int_{\Omega} w_{1} d \mathbf{x}=|\Omega|^{2} R_{m}\left(\mathbf{x}_{0}\right) / D$ from (2.1), we obtain

$$
\overline{\mathbb{T}}_{1}=\frac{|\Omega|^{2}}{2 \pi C_{0} D^{2}} R_{m}\left(\mathbf{x}_{0}\right)+4 \pi C_{0} \mathbb{T}_{0} R_{m}\left(\mathbf{x}_{0}\right)
$$

By substituting (2.17) into (2.12), and recalling (2.3), we obtain a two-term expansion for $\mathbb{T}$ in in the outer region

$$
\mathbb{T} \sim \frac{\mathbb{T}_{0}}{\varepsilon^{2}}+\frac{1}{\varepsilon}\left[-4 \pi C_{0} \mathbb{T}_{0} G_{m}\left(\mathbf{x} ; \mathbf{x}_{0}\right)+4 \pi C_{0} T_{0} R_{m}\left(\mathbf{x}_{0}\right)+\frac{|\Omega|^{2}}{2 \pi C_{0} D^{2}} R_{m}\left(\mathbf{x}_{0}\right)\right]+\mathcal{O}(1) .
$$

Finally, the variance of the first passage time $\mathcal{V}$ in the outer region is $\mathcal{V}=\mathbb{T}-w^{2}$, where $\mathbb{T}$ and $w$ are given in (2.18) and (2.1), respectively, while the standard deviation $\Sigma$ is $\Sigma=\mathcal{V}^{1 / 2}$. This leads to the following main result.

PRINCIPAL Result 2.2. In the limit $\varepsilon \rightarrow 0$ of small trap radius, the variance $\mathcal{V}$ and standard deviation $\Sigma=\mathcal{V}^{1 / 2}$ of the first passage time is given asymptotically in the outer region $\left|\mathbf{x}-\mathbf{x}_{0}\right| \gg \mathcal{O}(\varepsilon)$ by

$\mathcal{V} \sim \frac{|\Omega|^{2}}{16 \pi^{2} C_{0}^{2} D^{2} \varepsilon^{2}}+\frac{|\Omega|^{2}}{2 \pi C_{0} D^{2} \varepsilon} R_{m}\left(\mathbf{x}_{0}\right)+\mathcal{O}(1), \Sigma \sim \frac{|\Omega|}{4 \pi C_{0} D \varepsilon}\left[1+4 \pi \varepsilon C_{0} R_{m}\left(\mathbf{x}_{0}\right)+\mathcal{O}(\varepsilon)\right]$, where the regular part $R_{m}\left(\mathbf{x}_{0}\right)$ of the Green's function satisfies (1.3).

From (2.19) we conclude that the first two terms in the expansion of the variance and standard deviation are independent of the starting point for Brownian motion; only the $\mathcal{O}(1)$ term, which we did not calculate, depends on $\mathbf{x}$. Moreover, we conclude upon comparing (2.19) and (1.4) that the standard deviation of the first passage time is identical, up to $\mathcal{O}(1)$ terms, to the average MFPT $\tau$. This new result indicates that the average MFPT may not be a reliable estimate of the time for capture, as there is always a significant spread in the distribution about the mean.

The asymptotic equivalence of the mean and standard deviation of the capture time as $\varepsilon \rightarrow 0$ can be related to the long time form of the occupation density. Specifically, the probability $p(\mathbf{x}, t)$ that a random walker is free at $\mathbf{x} \in \Omega$ at time $t$ satisfies (cf. $[33,24,38,26]$ )

$$
p(\mathbf{x}, t) \propto \phi_{0}(\mathbf{x} ; \varepsilon) e^{-D \lambda_{0}(\varepsilon) t}, \quad t \rightarrow \infty,
$$

where $\left(\lambda_{0}(\varepsilon), \phi_{0}(\mathbf{x} ; \varepsilon)\right)$ is the principal eigenpair of the Laplacian in $\Omega \backslash \Omega_{\varepsilon}$. Therefore, Brownian particles that are not quickly absorbed have exponentially distributed capture times of equal mean and standard deviation.

To further illustrate this qualitative result between the MFPT and the standard deviation, suppose that the starting point for Brownian motion $\mathbf{x}$ is not far from $\mathbf{x}_{0}$. Then, since $G_{m}\left(\mathbf{x} ; \mathbf{x}_{0}\right)>0$, we have $w<\tau$, so that the MFPT is less than the average MFPT $\tau$. However, from (2.19), the asymptotic approximation of the 
standard deviation is not reduced when $\mathbf{x}$ becomes closer to $\mathbf{x}_{0}$, which presumably reflects the fact that although the particle may start relatively close to the trap, there are always some Brownian paths that miss the target and, thereby, avoid a quick capture.

Finally, we comment on the dependence of $\Sigma$ on $\mathbf{x}_{0}$. When $\Omega$ is a sphere of unit radius we have from [11] that

$$
R_{m}\left(\mathbf{x}_{0}\right)=\frac{1}{4 \pi\left(1-\left|\mathbf{x}_{0}\right|^{2}\right)}-\frac{1}{4 \pi} \log \left(1-\left|\mathbf{x}_{0}\right|^{2}\right)+\frac{\left|\mathbf{x}_{0}\right|^{2}}{4 \pi}-\frac{7}{10 \pi} .
$$

We obtain that $R_{m}\left(\mathbf{x}_{0}\right)$ is monotone increasing in $\left|\mathbf{x}_{0}\right|$, and so the standard deviation and the average MFPT are minimized, as expected, when the trap is centered at the origin of the sphere.

3. Determination of the capacitance of the structured target. In this section we use the method of matched asymptotic expansions to calculate the capacitance $C_{0}$, as defined in (1.5), when $\Omega_{0}$ is the unit sphere, and where the absorbing boundary set $\Gamma_{a}$ is subdivided into $N$ small patches, referred to here as nanotraps, each with radius $\sigma a_{k}$, where $\sigma \ll 1$. The centers of these boundary nanotraps are at $\mathbf{y}_{k}$, where $\left|\mathbf{y}_{k}\right|=1$ for $k=1, \ldots, N$. In this section, we refer to the small $\mathcal{O}(\sigma)$ neighborhood near the nanotrap as the "inner" region, while the region away from the nanotraps where $|\mathbf{y}|=\mathcal{O}(1)$ is referred to as the "outer" region. This result can then be used in Principal Results 2.1 and 2.2 to calculate the MFPT and the standard deviation of the capture times.

In terms of spherical coordinates $(r, \theta, \phi)$, the Laplacian in (1.5) is

$$
\Delta v \equiv \frac{\partial^{2} v}{\partial r^{2}}+\frac{2}{r} \frac{\partial v}{\partial r}+\frac{1}{r^{2} \sin ^{2} \theta} \frac{\partial^{2} v}{\partial \phi^{2}}+\frac{\cot \theta}{r^{2}} \frac{\partial v}{\partial \theta}+\frac{1}{r^{2}} \frac{\partial^{2} v}{\partial \theta^{2}} .
$$

In a boundary layer in the vicinity of the nanotrap centered at $\mathbf{y}_{k}$, we introduce the local coordinate system

$$
\mathbf{z}=\sigma^{-1}\left(\mathbf{y}-\mathbf{y}_{k}\right), \quad \eta=\sigma^{-1}(r-1), \quad s_{1}=\sigma^{-1} \sin \theta_{k}\left(\phi-\phi_{k}\right), \quad s_{2}=\sigma^{-1}\left(\theta-\theta_{k}\right),
$$

where $\eta$ is a rescaled measure of distance to the target and $\left(s_{1}, s_{2}\right)$ parameterizes the surface of the target for $\eta=0$. The particular form of $\left(s_{1}, s_{2}\right)$ is motivated by the surface distance metric. If $\mathbf{y}=(\sin \theta \cos \phi, \sin \theta \sin \phi, \cos \theta)^{T}$ and $\mathbf{y}_{k}=\left(\sin \theta_{k} \cos \phi_{k}, \sin \theta_{k} \sin \phi_{k}, \cos \theta_{k}\right)^{T}$ are two points on the sphere, then $\mathbf{y} \rightarrow \mathbf{y}_{k}$ implies that $(\phi, \theta) \rightarrow\left(\phi_{k}, \theta_{k}\right)$ and

$$
\begin{aligned}
\left|\mathbf{y}-\mathbf{y}_{k}\right|^{2} & =4 \sin \theta \sin \theta_{k} \sin ^{2}\left(\frac{\phi-\phi_{k}}{2}\right)+4 \sin ^{2}\left(\frac{\theta-\theta_{k}}{2}\right) \\
& \sim\left(\phi-\phi_{k}\right)^{2} \sin ^{2} \theta_{k}+\left(\theta-\theta_{k}\right)^{2}=\sigma^{2}\left[s_{1}^{2}+s_{2}^{2}\right] .
\end{aligned}
$$

Therefore $\left(s_{1}, s_{2}\right)$ is an approximate surface cartesian coordinate system near each nanotrap. In the local coordinates $(3.2)$, we define the operator $\mathcal{L}$ by $\mathcal{L} \equiv \partial_{\eta \eta}+$ $\partial_{s_{1} s_{1}}+\partial_{s_{2} s_{2}}$, and (3.1) becomes

$$
\Delta v \sim \sigma^{-2} \mathcal{L} v+\sigma^{-1}\left[-2 \eta\left(v_{s_{1} s_{1}}+v_{s_{2} s_{2}}\right)+\cot \theta_{k}\left(v_{s_{2}}-2 s_{2} v_{s_{1} s_{1}}\right)+2 v_{\eta}\right]+\mathcal{O}(1)
$$

As shown in Appendix A, the local behavior of the Green's function $G\left(\mathbf{y} ; \mathbf{y}_{k}\right)$ given in $(1.7 \mathrm{c})$ as $\mathbf{y} \rightarrow \mathbf{y}_{k}$, written in terms of the coordinates (3.2), is

$$
G=\frac{1}{2 \pi}\left[\frac{1}{\sigma|\mathbf{z}|}+\frac{1}{2} \log \left(\frac{\sigma}{2}\right)+\frac{1}{2} \log [\eta+|\mathbf{z}|]+\mathcal{O}(\sigma)\right] .
$$


Then, using (A.4) of Lemma A.1 of Appendix A, which gives a two-term expansion for $1 /|\mathbf{z}|$ in terms of the local coordinates $\left(s_{1}, s_{2}, \rho\right)$, where $\rho \equiv\left(s_{1}^{2}+s_{2}^{2}+\eta^{2}\right)^{1 / 2}$, we obtain that the local behavior of $G\left(\mathbf{y} ; \mathbf{y}_{k}\right)$ as $\mathbf{y} \rightarrow \mathbf{y}_{k}$ is

$$
G=\frac{1}{2 \pi}\left[\frac{1}{\sigma \rho}+\frac{1}{2} \log \left(\frac{\sigma}{2}\right)+\frac{1}{2}\left(\log [\eta+\rho]-\frac{1}{\rho^{3}}\left(\eta\left(s_{1}^{2}+s_{2}^{2}\right)+s_{1}^{2} s_{2} \cot \theta_{k}\right)\right)\right]+\mathcal{O}(\sigma) .
$$

In view of (3.5), we will expand the inner solution near the $k$ th nanotrap, in terms of the inner variables $\left(\rho, s_{1}, s_{2}\right)$, as

$$
v \sim \frac{w_{0}}{\sigma}+\log \left(\frac{\sigma}{2}\right) w_{1}+w_{2}+o(1) .
$$

Further terms in this inner expansion are generated systematically below. Since the nanotraps have radii $\mathcal{O}(\sigma)$, we anticipate that $C_{0}=\mathcal{O}(\sigma)$, and so in the outer region away from the nanotraps, we expect that $v \sim v_{0} / \sigma$, where $v_{0}$ is a constant. In this outer region, we expand the solution to (1.5) as

$$
v(\mathbf{y})=\frac{v_{0}}{\sigma}+v_{1}+\sigma \log \left(\frac{\sigma}{2}\right) v_{2}+\sigma v_{3}+\cdots,
$$

where $v_{0}$ is an unknown constant, and where each $v_{j}$ for $j \geq 1$ satisfies

$$
\Delta v_{j}=0, \quad|\mathbf{y}|>1 ; \quad \partial_{n} v_{j}=0, \quad \mathbf{y} \in \partial \Omega_{0} \backslash\left\{\mathbf{y}_{1}, \ldots, \mathbf{y}_{N}\right\}
$$

subject to certain asymptotic behaviors as $\mathbf{y} \rightarrow \mathbf{y}_{k}$ for $k=1, \ldots, N$ that are to be determined by matching. For each of these problems, we will show below that the solution will either be a constant or a superposition of Green's functions, where each nanotrap effectively introduces a Coulomb source of a certain strength. Further terms in the outer expansion are generated below, and we will need to adjust the outer expansion by certain logarithmic switchback terms (cf. [30]).

Upon substituting (3.6) into (1.5), and using (3.3), the leading-order problem from the $\mathcal{O}\left(\sigma^{-2}\right)$ terms is

$$
\mathcal{L} w_{0}=0, \quad \eta>0, \quad-\infty<s_{1}, s_{2}<\infty
$$

$$
w_{0}=0, \quad \eta=0, \quad s_{1}^{2}+s_{2}^{2}<a_{k}^{2} ; \quad \partial_{\eta} w_{0}=0, \quad \eta=0, \quad s_{1}^{2}+s_{2}^{2} \geq a_{k}^{2},
$$

subject to the matching condition that $w_{0} \sim v_{0}$ as $\rho \rightarrow \infty$. The solution to (3.9) is

$$
w_{0}=v_{0}\left(1-w_{c}\right),
$$

where $w_{c}$ is the solution to the electrified disk problem defined on the tangent plane to the sphere at $\mathbf{y}=\mathbf{y}_{k}$ by

$$
\mathcal{L} w_{c}=0, \quad \eta>0, \quad-\infty<s_{1}, s_{2}<\infty ; \quad w_{c} \rightarrow 0 \quad \text { as } \quad \rho \equiv \sqrt{s_{1}^{2}+s_{2}^{2}+\eta^{2}} \rightarrow \infty
$$

$$
w_{c}=1, \quad \eta=0, \quad s_{1}^{2}+s_{2}^{2}<a_{k}^{2} ; \quad \partial_{\eta} w_{c}=0, \quad \eta=0, \quad s_{1}^{2}+s_{2}^{2} \geq a_{k}^{2} .
$$

The exact solution to this problem (see page 38 of [19]) is (3.11c)

$w_{c}=\frac{2}{\pi} \sin ^{-1}\left(\frac{a_{k}}{L}\right), \quad L \equiv \frac{1}{2}\left[\sqrt{\left[\left(s_{1}^{2}+s_{2}^{2}\right)^{\frac{1}{2}}+a_{k}\right]^{2}+\eta^{2}}+\sqrt{\left[\left(s_{1}^{2}+s_{2}^{2}\right)^{\frac{1}{2}}-a_{k}\right]^{2}+\eta^{2}}\right]$. 
In terms of the capacitance $c_{k}=2 a_{k} / \pi$ of the $k$ th nanotrap, we readily obtain the far-field behavior

$$
w_{c} \sim c_{k}\left(\frac{1}{\rho}+\frac{\pi^{2} c_{k}^{2}}{24}\left(\frac{1}{\rho^{3}}-\frac{3 \eta^{2}}{\rho^{5}}\right)+\cdots\right) \quad \text { as } \quad \rho \rightarrow \infty ; \quad c_{k} \equiv \frac{2 a_{k}}{\pi} .
$$

Then, by using the far-field behavior (3.11d) together with the leading order behavior $\rho \sim \sigma^{-1}\left|\mathbf{y}-\mathbf{y}_{k}\right|$, we obtain that the near-field behavior as $\mathbf{y} \rightarrow \mathbf{y}_{k}$ of the outer expansion $\sigma^{-1} v_{0}+v_{1}+\cdots$ in (3.7) must agree with the far-field behavior of the inner expansion $\sigma^{-1} w_{0} \sim \sigma^{-1} v_{0}\left(1-\sigma c_{k} /\left|\mathbf{y}-\mathbf{y}_{k}\right|\right)$. In this way, we obtain that $v_{1}$ satisfies (3.8), subject to $v_{1} \sim-v_{0} c_{k} /\left|\mathbf{y}-\mathbf{y}_{k}\right|$ as $\mathbf{y} \rightarrow \mathbf{y}_{k}$ for $k=1, \ldots, N$. In terms of $G\left(\mathbf{y} ; \mathbf{y}_{0}\right)$ satisfying (1.7), the solution for $v_{1}$ is

$$
v_{1}=-2 \pi v_{0} \sum_{j=1}^{N} c_{j} G\left(\mathbf{y} ; \mathbf{y}_{j}\right)+\chi_{1},
$$

where $\chi_{1}$ is a constant to be determined. Now for $|\mathbf{y}| \gg 1$, we have from Appendix A that $G\left(\mathbf{y} ; \mathbf{y}_{j}\right) \sim(4 \pi|\mathbf{y}|)^{-1}$. Therefore, from the prescribed flux condition defined by (1.5b), we determine the unknown constant $v_{0}$ as

$$
v_{0}=\frac{-2}{\sum_{j=1}^{N} c_{j}}=\frac{-2}{N \bar{c}}, \quad \text { where } \quad \bar{c} \equiv \frac{1}{N} \sum_{j=1}^{N} c_{j} .
$$

To proceed to higher order, we expand $v_{1}$ in (3.12) as $\mathbf{y} \rightarrow \mathbf{y}_{k}$, by using the local behavior (3.5), to obtain that

$$
\begin{aligned}
\frac{v_{0}}{\sigma}+v_{1}+\cdots \sim \frac{v_{0}}{\sigma}\left(1-\frac{c_{k}}{\rho}\right)-v_{0} c_{k}[ & \frac{1}{2} \log \left(\frac{\sigma}{2}\right)+\frac{1}{2} \log [\eta+\rho] \\
& \left.-\frac{1}{2 \rho^{3}}\left(\eta\left(s_{1}^{2}+s_{2}^{2}\right)+s_{1}^{2} s_{2} \cot \theta_{k}\right)\right]+\chi_{1}+B_{k},
\end{aligned}
$$

where we have defined the constant $B_{k}$ by

$$
B_{k}=-2 \pi v_{0} \sum_{\substack{j=1 \\ j \neq k}}^{N} c_{j} G\left(\mathbf{y}_{k} ; \mathbf{y}_{j}\right), \quad k=1, \ldots, N .
$$

This shows that the next term in the inner expansion is $\mathcal{O}(\log \sigma)$, as written in (3.6). To shed light on how the particular spatial arrangement of nanotraps affects capture, we require a high order expansion which incorporates the interaction terms $B_{k}$. In order to obtain a well-posed problem for $v_{2}$, where we have a degree of freedom to impose the zero flux condition $\lim _{R \rightarrow \infty} \int_{\partial \Omega_{R}} \partial_{n} v_{2} d s=0$, we must incorporate a constant logarithmic switchback term (cf. [30]) of order $\mathcal{O}(\log \sigma)$ into the outer expansion. This is equivalent to decomposing the unknown $\chi_{1}$ in (3.12) as

$$
\chi_{1}=\chi_{10}\left(\log \frac{\sigma}{2}\right)+\chi_{11} .
$$

As such, with the inclusion of this switchback term, our modified outer expansion becomes

$$
v(\mathbf{y})=\frac{v_{0}}{\sigma}+\chi_{10} \log \left(\frac{\sigma}{2}\right)+v_{1}+\sigma \log \left(\frac{\sigma}{2}\right) v_{2}+\sigma v_{3}+\cdots
$$


where

$$
v_{1}=-2 \pi v_{0} \sum_{j=1}^{N} c_{j} G\left(\mathbf{y} ; \mathbf{y}_{j}\right)+\chi_{11} .
$$

Upon using (3.15) for $\chi_{1}$ in (3.14a) and comparing this with (3.6), we obtain using (3.3) that $w_{1}$ satisfies (3.9) subject to the far-field behavior that $w_{1} \sim\left(\chi_{10}-\frac{c_{k} v_{0}}{2}\right)$ as $\rho \rightarrow \infty$. As such, the exact solution for $w_{1}$ is

$$
w_{1}=\left(\chi_{10}-\frac{c_{k} v_{0}}{2}\right)\left(1-w_{c}\right),
$$

where $w_{c}$ is the solution (3.11c) to the electrified disk problem (3.11). By using the far-field behavior (3.11d), we then match the monopole behavior for $w_{1}$ to the term $v_{2}$ in the outer expansion (3.16). In this way, we find that $v_{2}$ satisfies (3.8) subject to the singularity behavior $v_{2} \sim-c_{k}\left(\chi_{10}-c_{k} v_{0} / 2\right) /\left|\mathbf{y}-\mathbf{y}_{k}\right|$ as $\mathbf{y} \rightarrow \mathbf{y}_{k}$ for $k=1, \ldots, N$. In terms of a further unknown constant $\chi_{2}$, the solution for $v_{2}$ is

$$
v_{2}=-2 \pi \sum_{j=1}^{N} c_{k}\left(\chi_{10}-\frac{c_{k} v_{0}}{2}\right) G\left(\mathbf{y} ; \mathbf{y}_{j}\right)+\chi_{2} .
$$

Then, by imposing the no-flux condition $\lim _{R \rightarrow \infty} \int_{\partial \Omega_{R}} \partial_{n} v_{2} d s=0$, we get $\sum_{j=1}^{N} c_{j}\left(\chi_{10}\right.$ $\left.-\frac{c_{j} v_{0}}{2}\right)=0$, which yields that

$$
\chi_{10}=\frac{v_{0}}{2 N \bar{c}} \sum_{k=1}^{N} c_{k}^{2}=-\frac{1}{(N \bar{c})^{2}} \sum_{k=1}^{N} c_{k}^{2} .
$$

Then, similarly to the discussion above regarding $v_{1}$, we must decompose $\chi_{2}$ in (3.19) as

$$
\chi_{2}=\chi_{20} \log \left(\frac{\sigma}{2}\right)+\chi_{21}
$$

which effectively introduces a further constant switchback term $\sigma[\log (\sigma / 2)]^{2} \chi_{20}$ into the outer expansion, yielding

$$
v(\mathbf{y})=\frac{v_{0}}{\sigma}+\chi_{10} \log \left(\frac{\sigma}{2}\right)+v_{1}+\sigma\left[\log \left(\frac{\sigma}{2}\right)\right]^{2} \chi_{20}+\sigma \log \left(\frac{\sigma}{2}\right) v_{2}+\sigma v_{3}+\cdots
$$

where $v_{2}$ is given by

$$
v_{2}=-2 \pi \sum_{j=1}^{N} c_{k}\left(\chi_{10}-\frac{c_{k} v_{0}}{2}\right) G\left(\mathbf{y} ; \mathbf{y}_{j}\right)+\chi_{21} .
$$

We will calculate $\chi_{20}$ explicitly below, while the term $\chi_{21}$ will be used only to provide our error estimate for $C_{0}$.

The intricate step in the analysis is to determine $\chi_{11}$ in (3.17) which incorporates the self-interaction terms $B_{k}$ given in (3.14b). This is done by equating the far-field behavior of $w_{2}$ as $\rho \rightarrow \infty$ in the inner expansion (3.6) with the $\mathcal{O}(1)$ terms in the near-field behavior (3.14a) for $v_{1}$. This yields that

$w_{2} \sim-v_{0} c_{k}\left[\frac{1}{2} \log [\eta+\rho]-\frac{1}{2 \rho^{3}}\left(\eta\left(s_{1}^{2}+s_{2}^{2}\right)+s_{1}^{2} s_{2} \cot \theta_{k}\right)\right]+\chi_{11}+B_{k} \quad$ as $\quad \rho \rightarrow \infty$. 
Moreover, from substituting the inner expansion (3.6) into (3.3), and then using $w_{0}=v_{0}\left(1-w_{c}\right)$ to rewrite the inhomogeneous terms in the resulting PDE, we obtain that $w_{2}$ satisfies the Poisson-type problem

(3.24b)

$\mathcal{L} w_{2}=2 v_{0}\left(w_{c \eta}+\eta w_{c \eta \eta}\right)+v_{0} \cot \theta_{k}\left(w_{c s_{2}}-2 s_{2} w_{c s_{1} s_{1}}\right), \quad \eta>0, \quad-\infty<s_{1}, s_{2}<\infty$,

$$
w_{2}=0, \quad \eta=0, \quad s_{1}^{2}+s_{2}^{2}<a_{k}^{2} ; \quad \partial_{\eta} w_{2}=0, \quad \eta=0, \quad s_{1}^{2}+s_{2}^{2} \geq a_{k}^{2},
$$

subject to the far-field behavior (3.24a) for $w_{2}$ as $\rho \rightarrow \infty$.

The analysis for $w_{2}$ is very similar to that done in [12] in the context of the narrow escape problem, with the exception that $\eta$ has a different sign here since we are solving exterior to the target sphere, rather than interior to a confining sphere. In Appendix $\mathrm{B}$ we show that the homogeneous part of the solution for $w_{2}$ is determined by the $\mathcal{O}(1)$ terms in the far-field behavior (3.24a), while the remaining terms match particular solutions arising from certain inhomogeneous terms on the right-hand side of (3.24b). As summarized in Appendix B, the appropriate decomposition for $w_{2}$ is

$$
w_{2}=\left(\chi_{11}+B_{k}\right)\left(1-w_{c}\right)+v_{0} w_{2 o}+v_{0} w_{2 e},
$$

where $w_{c}$ satisfies (3.11). Here $w_{2 o}$ is the solution to

$$
\mathcal{L} w_{2 o}=\cot \theta_{k}\left(w_{c s_{2}}-2 s_{2} w_{c s_{1} s_{1}}\right), \quad \eta>0, \quad-\infty<s_{1}, s_{2}<\infty
$$

$$
w_{2 o}=0, \quad \eta=0, \quad s_{1}^{2}+s_{2}^{2}<a_{k}^{2} ; \quad \partial_{\eta} w_{2 o}=0, \quad \eta=0, \quad s_{1}^{2}+s_{2}^{2} \geq a_{k}^{2},
$$

$$
w_{2 o} \sim \frac{c_{k}}{2 \rho^{3}}\left(s_{1}^{2} s_{2} \cot \theta_{k}\right) \quad \text { as } \quad \rho \rightarrow \infty
$$

while $w_{2 e}$ satisfies

$$
\mathcal{L} w_{2 e}=2\left(w_{c \eta}+\eta w_{c \eta \eta}\right), \quad \eta>0, \quad-\infty<s_{1}, s_{2}<\infty,
$$

$$
\begin{aligned}
& w_{2 e}=0, \quad \eta=0, \quad s_{1}^{2}+s_{2}^{2}<a_{k}^{2} ; \quad \partial_{\eta} w_{2 e}=0, \quad \eta=0, \quad s_{1}^{2}+s_{2}^{2} \geq a_{k}^{2}, \\
& w_{2 e} \sim-\frac{c_{k}}{2} \log [\eta+\rho]+\frac{c_{k}}{2 \rho^{3}} \eta\left(s_{1}^{2}+s_{2}^{2}\right) \quad \text { as } \quad \rho \rightarrow \infty .
\end{aligned}
$$

The key issue in the analysis of $w_{2}$ is to identify which terms in the decomposition (3.25) can generate monopole terms of the form $a / \rho$ in the far-field behavior as $\rho \rightarrow \infty$. Such terms will contribute to the determination of $\chi_{11}$ from a solvability condition applied to the problem for the outer correction $v_{3}$ in (3.16). Clearly, the first term $\left(\chi_{11}+B_{k}\right)\left(1-w_{c}\right)$ in $(3.25)$ yields a monopole term from the far-field behavior of $w_{c}$ given in (3.11d). However, by solving the problems for $w_{2 e}$ and $w_{2 o}$ exactly as in Lemmas B.1 and B.2 of Appendix B, respectively, we find that of these two terms only $w_{2 e}$ yields a monopole term, and it has the far-field behavior (see (B.4) of Appendix B)

$$
\begin{gathered}
w_{2 e}=-\frac{c_{k}}{2} \log [\eta+\rho]+\frac{c_{k}}{2 \rho^{3}} \eta\left(s_{1}^{2}+s_{2}^{2}\right)+\frac{c_{k} b_{k}}{\rho}+\mathcal{O}\left(\rho^{-2}\right) \quad \text { as } \quad \rho \rightarrow \infty, \\
\text { where } \quad b_{k}=\frac{c_{k}}{2}\left[\log \left(4 a_{k}\right)-\frac{3}{2}\right] .
\end{gathered}
$$


In this way, the far-field behavior of $w_{2}$ from (3.25) is

$$
\begin{gathered}
w_{2} \sim-v_{0} c_{k}\left[\frac{1}{2} \log [\eta+\rho]-\frac{1}{2 \rho^{3}}\left(\eta\left(s_{1}^{2}+s_{2}^{2}\right)+s_{1}^{2} s_{2} \cot \theta_{k}\right)\right]+\chi_{11}+B_{k}-\frac{c_{k}\left(\chi_{11}+B_{k}-v_{0} b_{k}\right)}{\rho}, \\
\text { as } \rho \rightarrow \infty
\end{gathered}
$$

Since $\rho \sim \sigma^{-1}\left|\mathbf{y}-\mathbf{y}_{k}\right|$, the monopole term for $w_{2}$ generates a singularity behavior for the correction term $v_{3}$ in the outer expansion (3.16). We find that $v_{3}$ satisfies (3.8) subject to the singularity behavior $v_{3} \sim-c_{k}\left(\chi_{11}+B_{k}-v_{0} b_{k}\right) /\left|\mathbf{y}-\mathbf{y}_{k}\right|$ as $\mathbf{y} \rightarrow \mathbf{y}_{k}$ for $k=1, \ldots, N$. By imposing the no far-field flux condition $\lim _{R \rightarrow \infty} \int_{\partial \Omega_{R}} \partial_{n} v_{3} d s=0$, we determine $\chi_{11}$ as

$$
\chi_{11}=\frac{1}{N \bar{c}} \sum_{k=1}^{N} c_{k}\left(v_{0} b_{k}-B_{k}\right),
$$

where $B_{k}$ is defined in (3.14b). Upon substituting (3.14b) into (3.30), we determine $\chi_{11}$ as

$$
\begin{aligned}
\chi_{11}= & \frac{v_{0}}{N \bar{c}}\left[\sum_{k=1}^{N} c_{k} b_{k}+2 \mathcal{F}\left(\mathbf{y}_{1}, \ldots, \mathbf{y}_{N}\right)\right] \\
& \text { where } \mathcal{F}\left(\mathbf{y}_{1}, \ldots, \mathbf{y}_{N}\right) \equiv 2 \pi \sum_{j=1}^{N} \sum_{k=j+1}^{N} c_{j} c_{k} G\left(\mathbf{y}_{j} ; \mathbf{y}_{k}\right) .
\end{aligned}
$$

Here we have used the reciprocity relation $G\left(\mathbf{y}_{j} ; \mathbf{y}_{k}\right)=G\left(\mathbf{y}_{k} ; \mathbf{y}_{j}\right)$ of the Green's function $(1.7 \mathrm{c})$.

Finally, similarly to the determination of $\chi_{10}$, we can calculate the constant $\chi_{20}$ in (3.21). This calculation, not performed for the narrow escape problem in [12], requires the introduction of further correction terms in the inner and outer expansions. The modified outer expansion is now given by

$$
\begin{aligned}
v(\mathbf{y})= & \frac{v_{0}}{\sigma}+\chi_{10} \log \left(\frac{\sigma}{2}\right)+v_{1}+\sigma\left[\log \left(\frac{\sigma}{2}\right)\right]^{2} \chi_{20}+\sigma \log \left(\frac{\sigma}{2}\right) v_{2} \\
& +\sigma v_{3}+\sigma^{2}\left[\log \left(\frac{\sigma}{2}\right)\right]^{2} v_{4}+\cdots,
\end{aligned}
$$

while the modified form of the inner expansion is

$$
v \sim \frac{w_{0}}{\sigma}+\log \left(\frac{\sigma}{2}\right) w_{1}+w_{2}+\sigma\left[\log \left(\frac{\sigma}{2}\right)\right]^{2} w_{3}+\cdots .
$$

The $\mathcal{O}(\log \sigma)$ local behavior of $v_{2}$, together with the switchback term in (3.32) proportional to $\chi_{20}$, yields that $w_{3}$ must have the far-field behavior $w_{3} \sim \chi_{20}-\frac{c_{k}}{2}\left(\chi_{10}-\frac{v_{0} c_{k}}{2}\right)$ as $\rho \rightarrow \infty$. Then, upon substituting (3.33) into (3.1), we obtain that $\mathcal{L} w_{3}=0$, so that

$$
w_{3} \equiv\left[\chi_{20}-\frac{c_{k}}{2}\left(\chi_{10}-\frac{v_{0} c_{k}}{2}\right)\right]\left(1-w_{c}\right) .
$$

By using $w_{c} \sim c_{k} / \rho$ as $\rho \rightarrow \infty$, the far-field behavior of $w_{3}$ then yields a singularity condition for the outer correction $v_{4}$ in (3.32). We find that $v_{4}$ satisfies (3.8), subject 
to the singularity condition

$$
v_{4} \sim-\frac{c_{k}\left[\chi_{20}-\frac{c_{k}}{2}\left(\chi_{10}-\frac{v_{0} c_{k}}{2}\right)\right]}{\left|\mathbf{y}-\mathbf{y}_{k}\right|} \quad \text { as } \quad \mathbf{y} \rightarrow \mathbf{y}_{k}, \quad k=1, \ldots, N .
$$

Then, by imposing the no far-field flux condition $\lim _{R \rightarrow \infty} \int_{\partial \Omega_{R}} \partial_{n} v_{4} d s=0$, we get $2 \chi_{20} \sum_{k=1}^{N} c_{k}=\sum_{k=1}^{N}\left(\chi_{10}-\frac{v_{0} c_{k}}{2}\right) c_{k}^{2}$. By using (3.20) for $\chi_{10}$, we determine $\chi_{20}$ as

$$
\chi_{20}=\frac{v_{0}}{4 N \bar{c}}\left(\frac{\left(\sum_{k=1}^{N} c_{k}^{2}\right)^{2}}{N \bar{c}}-\sum_{k=1}^{N} c_{k}^{3}\right) .
$$

Finally, to identify the capacitance $C_{0}$ we let $|\mathbf{y}| \rightarrow \infty$ in (3.32) and compare the resulting expression with (1.5c). Since $\lim _{R \rightarrow \infty} \int_{\partial \Omega_{R}} \partial_{n} v_{j} d s=-4 \pi \delta_{j, 1}$, where $\delta_{j, 1}$ is the Kronecker symbol, we readily identify that

$$
-\frac{1}{C_{0}}=\frac{v_{0}}{\sigma}+\chi_{10} \log \left(\frac{\sigma}{2}\right)+\chi_{11}+\sigma\left[\log \left(\frac{\sigma}{2}\right)\right]^{2} \chi_{20}+\mathcal{O}\left(\sigma \log \left(\frac{\sigma}{2}\right)\right) .
$$

Upon using our previous results for $v_{0}, \chi_{10}, \chi_{11}$, and $\chi_{20}$ from (3.13), (3.20), (3.31), and (3.36), we obtain our main result for the capacitance $C_{0}$ of the structured spherical target.

Principal Result 3.1. In the limit $\sigma \rightarrow 0$, the capacitance $C_{0}$, defined in (1.5), for the target unit sphere that contains $N$ nonoverlapping locally circular nanotraps of radii $\sigma a_{k}$ for $k=1, \ldots, N$, centered at $\mathbf{y}_{k}$ for $k=1, \ldots, N$ on its boundary, is given asymptotically by

$$
\begin{aligned}
\frac{1}{C_{0}}=\frac{2}{N \bar{c} \sigma}[1 & +\sigma \log \left(\frac{\sigma}{2}\right) \frac{\sum_{j=1}^{N} c_{j}^{2}}{2 N \bar{c}}+\frac{\sigma}{N \bar{c}}\left(\sum_{k=1}^{N} c_{k} b_{k}+2 \mathcal{F}\left(\mathbf{y}_{1}, \ldots, \mathbf{y}_{N}\right)\right) \\
& \left.+\sigma^{2}\left[\log \left(\frac{\sigma}{2}\right)\right]^{2} \frac{\zeta}{4 N \bar{c}}+\mathcal{O}\left(\sigma^{2} \log \left(\frac{\sigma}{2}\right)\right)\right]
\end{aligned}
$$

Here $c_{k}=2 a_{k} / \pi$ is the capacitance of the $k$ th circular nanotrap, while $b_{k}, \zeta$, and $\mathcal{F}\left(\mathbf{y}_{1}, \ldots, \mathbf{y}_{N}\right)$ are defined by

$$
\begin{array}{r}
b_{k} \equiv \frac{c_{k}}{2}\left(\log \left(4 a_{k}\right)-\frac{3}{2}\right), \quad \zeta \equiv \frac{\left(\sum_{k=1}^{N} c_{k}^{2}\right)^{2}}{N \bar{c}}-\sum_{k=1}^{N} c_{k}^{3}, \\
\mathcal{F}\left(\mathbf{y}_{1}, \ldots, \mathbf{y}_{N}\right) \equiv 2 \pi \sum_{j=1}^{N} \sum_{k=j+1}^{N} c_{j} c_{k} G\left(\mathbf{y}_{j} ; \mathbf{y}_{k}\right)
\end{array}
$$

where the Green's interaction term $G\left(\mathbf{y}_{j} ; \mathbf{y}_{k}\right)$ can be calculated using (1.7c).

As a remark, we can use the more refined far-field behavior of $G\left(\mathbf{y} ; \mathbf{y}_{0}\right)$ given in (A.8) to calculate the leading order behavior in $\sigma$ of the dipole term $\mathbf{p}$ in (1.5c). However, since this term was not needed in section 2 we forgo this calculation.

In (3.37a), the discrete energy $\mathcal{F}\left(\mathbf{y}_{1}, \ldots, \mathbf{y}_{N}\right)$ depends on the spatial distribution of the nanotraps and contributes to $C_{0}$ a weighted sum of the Green's interaction term $G\left(\mathbf{y}_{j} ; \mathbf{y}_{k}\right)$. As two nanotraps become closer, the term $G\left(\mathbf{y}_{j} ; \mathbf{y}_{k}\right)$ increases, which 
then has the effect of decreasing $C_{0}$. We remark that the term $\zeta$ in (3.37a) vanishes when the nanotraps have a common radii, and so effectively it measures the spread in the distribution of the sizes of the nanotraps.

For the special case where $a_{k}=1$ for $k=1, \ldots, N$, in which the nanotraps have a common radius $\sigma$, we simply set $c_{k}=2 / \pi$ and $\zeta=0$ in (3.37). Then (3.37a) reduces to

$\frac{1}{C_{0}}=\frac{\pi}{N \sigma}\left[1+\frac{\sigma}{\pi} \log \left(\frac{\sigma}{2}\right)+\frac{\sigma}{\pi}\left(\log 4-\frac{3}{2}+\frac{4}{N} \mathcal{H}\left(\mathbf{y}_{1}, \ldots, \mathbf{y}_{N}\right)\right)+\mathcal{O}\left(\sigma^{2} \log \left(\frac{\sigma}{2}\right)\right)\right]$,

where the interaction function $\mathcal{H}\left(\mathbf{y}_{1}, \ldots, \mathbf{y}_{N}\right)$ defined by $\mathcal{F}\left(\mathbf{y}_{1}, \ldots, \mathbf{y}_{N}\right)=c_{k}^{2} \mathcal{H}\left(\mathbf{y}_{1}, \ldots\right.$, $\left.\mathbf{y}_{N}\right)$ is

$$
\mathcal{H}\left(\mathbf{y}_{1}, \ldots, \mathbf{y}_{N}\right)=\sum_{j=1}^{N} \sum_{k=j+1}^{N}\left[\frac{1}{\left|\mathbf{y}_{j}-\mathbf{y}_{k}\right|}+\frac{1}{2} \log \left|\mathbf{y}_{j}-\mathbf{y}_{k}\right|-\frac{1}{2} \log \left(2+\left|\mathbf{y}_{j}-\mathbf{y}_{k}\right|\right)\right] .
$$

For the special case $N=1$, the expression (3.38a) simplifies to

$$
\frac{1}{C_{0}}=\frac{\pi}{\sigma}\left[1+\frac{\sigma}{\pi} \log \left(\frac{\sigma}{2}\right)+\frac{\sigma}{\pi}\left(\log 4-\frac{3}{2}\right)+\mathcal{O}\left(\sigma^{2} \log \left(\frac{\sigma}{2}\right)\right)\right] .
$$

As a partial confirmation of our results, in section 6 we derive a high order asymptotic expansion of an exact solution for the single nanotrap case $N=1$, which yields that (3.40)

$$
\frac{1}{C_{0}}=\frac{\pi}{\sigma}\left[1+\frac{\sigma}{\pi}\left(\log \sigma-\frac{3}{2}+\log 2\right)-\frac{\sigma^{2}}{\pi^{2}}\left(\frac{\pi^{2}+21}{36}\right)+\mathcal{O}\left(\sigma^{3} \log \sigma\right)\right] \quad \text { as } \sigma \rightarrow 0
$$

The expression in Principal Result 3.1 and (3.38) are two of the main results of this paper. In section 4 we use (3.38) to derive a new effective Robin boundary condition on the sphere that corresponds to the homogenized limit $N=\mathcal{O}(-\log \sigma) \gg 1$, where the nanotraps are uniformly distributed over the surface of the sphere. In section 4.1 we use (3.38) to numerically explore the effect on $C_{0}$ of both the fragmentation of the nanotrap set and of the spatial distribution of the nanotraps on the boundary of the target sphere.

To validate the asymptotic formula (3.38) for the capacitance of the spherical target with multiple nanotraps and (3.39) for a single nanotrap, we compare these asymptotic results with full numerical solutions of the exterior problem (1.5), as obtained by means of a spectral boundary element method (cf. section 5).

4. The effective Robin boundary condition: A scaling law for large $N$. In this section we use our main result (3.38) to derive a scaling law for $1 / C_{0}$ for the case where $N=\mathcal{O}(-\log \sigma) \gg 1$ nanotraps are equally distributed over the surface of the sphere. In this low trap surface area fraction limit, we will derive from first principles a "homogenized" effective boundary condition of the form $\partial_{n} v+\kappa v=0$ to be used on $\partial \Omega_{0}$, for some $\kappa$ to be determined. Our result is then compared with previous results in the literature. We remark that in the context of the nuclear-pore structure, the nuclear radius is roughly 4 microns, and there are estimated to be approximately $N=2000$ nanotraps, each of estimated radius 25 nanometers (cf. [29, 41]). This yields that $\sigma=6.25 \times 10^{-3}$, and a surface area fraction $f$ of $f=N \sigma^{2} / 4 \approx 0.0195$, providing only a small $2 \%$ coverage of the boundary of the nucleus by nanotraps. 


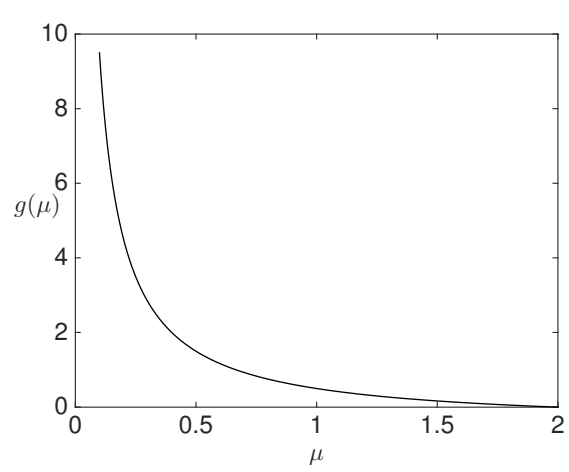

(a) Interaction energy $g(\mu)$.

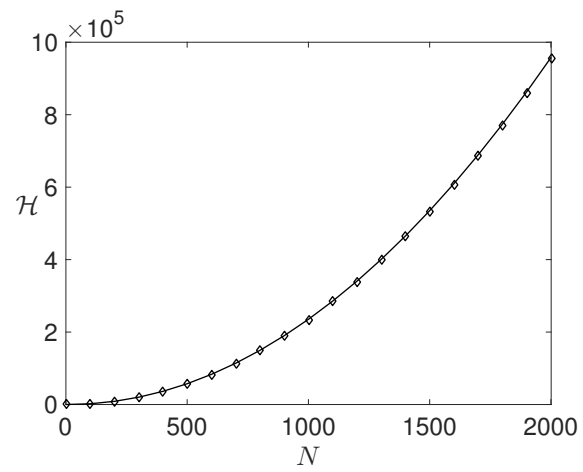

(b) Discrete energy $\mathcal{H}$.

FIG. 2. Left: Plot of the interaction energy $g(\mu)$ of (4.1a). Right: The discrete energy $\mathcal{H}$ of (4.1) for equally spaced Fibonacci points (diamonds) and the homogenized result (solid line) given in (4.4).

We first consider some properties of the discrete energy in (3.38b). Since $0<$ $\left|\mathbf{y}_{j}-\mathbf{y}_{k}\right| \leq 2$ for $j \neq k$, we have

$\mathcal{H}\left(\mathbf{y}_{1}, \ldots, \mathbf{y}_{N}\right)=\sum_{j=1}^{N} \sum_{k=j+1}^{N} g\left(\left|\mathbf{y}_{j}-\mathbf{y}_{k}\right|\right), \quad$ where $g(\mu) \equiv \frac{1}{\mu}+\frac{1}{2} \log \left(\frac{\mu}{2+\mu}\right), 0<\mu \leq 2$,

where $g(\mu)$ on $0<\mu<2$, with $g(\mu) \rightarrow+\infty$ as $\mu \rightarrow 0$, satisfies

$$
g^{\prime}(\mu)=-\frac{2}{\mu^{2}(2+\mu)}<0, \quad g^{\prime \prime}(\mu)=\frac{2(3 \mu+4)}{\mu^{3}(2+\mu)^{2}}>0 .
$$

Since $g(2)>0$, we conclude that $g(\mu)$ is a positive, monotone decreasing, and convex function on $0<\mu \leq 2$ (see Figure 2(a)). This indicates that the optimal configuration $\left\{\mathbf{y}_{1}, \ldots, \mathbf{y}_{N}\right\}$ of nanotraps that minimizes $\mathcal{H}\left(\mathbf{y}_{1}, \ldots, \mathbf{y}_{N}\right)$ should be, in some sense, "uniformly distributed" over the sphere, in order that the nanotraps be as far apart as possible. From (3.38a), it follows that the capacitance $C_{0}$ is maximized, and the corresponding average MFPT from (1.4) minimized, at the specific nanotrap configuration $\left\{\mathbf{y}_{1}, \ldots, \mathbf{y}_{N}\right\}$ that globally minimizes the discrete energy in (4.1a).

Since we anticipate that the optimal nanotrap configuration will be uniformly spaced over the sphere, we seek a scaling law for $\mathcal{H}$ as $N \rightarrow \infty$ for such a uniformly distributed arrangement. This scaling law is readily derived using the mean-field approximation method of [20] and [5]. Since a full discussion of this method was also given in [12] (see also [13]), we only give the main results here. For this scaling law, it is convenient to decompose $\mathcal{H}$ into three terms as

$$
\mathcal{H}\left(\mathbf{y}_{1}, \ldots, \mathbf{y}_{N}\right)=\mathcal{H}_{1}-\mathcal{H}_{2}+\mathcal{H}_{3}
$$

where

$$
\begin{array}{r}
\mathcal{H}_{1} \equiv \sum_{j=1}^{N} \sum_{k=j+1}^{N} \frac{1}{\left|\mathbf{y}_{j}-\mathbf{y}_{k}\right|}, \quad \mathcal{H}_{2} \equiv-\frac{1}{2} \sum_{j=1}^{N} \sum_{k=j+1}^{N} \log \left|\mathbf{y}_{j}-\mathbf{y}_{k}\right| \\
\mathcal{H}_{3} \equiv-\frac{1}{2} \sum_{j=1}^{N} \sum_{k=j+1}^{N} \log \left(2+\left|\mathbf{y}_{j}-\mathbf{y}_{k}\right|\right) .
\end{array}
$$


The expression in (4.2) differs from that for the narrow escape problem [12] by the sign of the middle term in (4.2a), so that, in contrast to [12], $\mathcal{H}$ is no longer the sum of three convex energies. We remark that the classic discrete variational problems of minimizing either the Coulomb energy $\mathcal{H}_{1}$ or the logarithmic energy $\mathcal{H}_{2}$ on the unit sphere have a long history in approximation theory (see $[20,5,39,27,44,7]$ ). For these energies, the optimal configurations for large $N$ are hexagonal patterns, with some defects consisting of pentagonal faces that are needed to tessellate the sphere.

For a uniform distribution of points on the sphere, we have for large $N$ that (cf. [12])

$$
\begin{aligned}
& \mathcal{H}_{1}=\frac{1}{2} N^{2}-\frac{1}{2} N^{3 / 2}+\frac{1}{12} N^{1 / 2}+o(1), \\
& \mathcal{H}_{2}=\frac{N^{2}}{8}(1-\log 4)-\frac{1}{8} N \log N-\frac{1}{8} N(1-\log 4)+\mathcal{O}(\log N), \\
& \mathcal{H}_{3}=-\frac{N^{2}}{8}(1+\log 4)+\frac{\log 2}{4} N+\frac{N^{1 / 2}}{6}+\mathcal{O}(1),
\end{aligned}
$$

which from (4.2a) yields the following formal scaling law for uniformly distributed nanotraps as $N \rightarrow \infty$ :

$$
\mathcal{H}=\frac{N^{2}}{4}-\frac{1}{2} N^{3 / 2}+\frac{1}{8} N \log N+\frac{N}{8}+\frac{N^{1 / 2}}{4}+\mathcal{O}(\log N) .
$$

However, since this mean-field approximation completely disregards the details of the spatial distribution of points on the sphere, it does not provide a reliable scaling law for the true globally minimum energy configuration. The highest powers of $N$ obtained by this approximation are presumably theoretically correct in analogy with previous rigorous results for the classical Coulomb $\mathcal{H}_{1}$ or logarithmic $\mathcal{H}_{2}$ energies (see [27] and [39]). However, the coefficients of the lower order terms should depend on the details of the optimal nanotrap arrangement. In particular, from a numerical computation of $N \leq 200$ particles, in [39] it was shown that the optimal energy for $\mathcal{H}_{1}$ on the sphere can be well fitted with

$$
\mathcal{H}_{1 \min } \approx \frac{N^{2}}{2}-0.55230 N^{3 / 2}+0.0689 N^{1 / 2} .
$$

As emphasized in [20], the coefficient of $N^{3 / 2}$ should be essentially independent of whether the points are arranged on a sphere or on a flat plane. Therefore, neglecting the minor effect due to defects in the hexagonal point pattern on the sphere, the coefficient of $N^{3 / 2}$ should be very closely approximated by that for a hexagonal arrangement of points on the flat plane. Regarding the optimality of $\mathcal{H}_{2}$, it was proved in [27] that the coefficients of $N^{2}$ and the $N \log N$ in the formal scaling law hold for the optimal $\mathcal{H}_{2}$, and it was conjectured in [39] that the next term is proportional to $N$. This has been proved recently in Theorem 1.2 of [44].

Based on these qualitative remarks, and in the absence of any rigorous results for $\mathcal{H}$, we postulate in terms of some unknown coefficients $d_{1}, d_{2}$, and $d_{3}$ that the optimal energy $\mathcal{H}_{\min }$ for $N \rightarrow \infty$ has the form

$$
\mathcal{H}_{\text {min }}=\frac{N^{2}}{4}-d_{1} N^{3 / 2}+\frac{1}{8} N \log N+d_{2} N+d_{3} N^{1 / 2}+\mathcal{O}(\log N)
$$

for some $d_{1}, d_{2}$, and $d_{3}$ with $d_{1} \approx 0.55230$. If we simply assume a uniform distribution of the centers of the nanotraps on the sphere, we have from (4.4) that $d_{1}=1 / 2$, 
$d_{2}=1 / 8$, and $d_{3}=1 / 4$. A comparison of (4.6) with the discrete energy in (4.1a) is shown in Figure 2(b).

Upon substituting (4.6) into (3.38a) we obtain that

$$
\frac{1}{C_{0}} \sim \frac{\pi}{N \sigma}+\frac{1}{N} \log \left(2 e^{-3 / 2} \sigma\right)+1-\frac{4 d_{1}}{\sqrt{N}}+\frac{1}{2 N} \log \left(N e^{8 d_{2}}\right)+\frac{4 d_{3}}{N^{3 / 2}} .
$$

In order to preserve the asymptotic ordering consistent with our derivation of (3.38), we need to assume that $N \leq \mathcal{O}(-\log \sigma)$, so that, at worst, the second and third terms on the right-hand side of (4.7) are of comparable order. With this assumption, (4.7) can be written as

$$
\frac{1}{C_{0}} \sim \frac{\pi}{N \sigma}+1+\frac{\log \left(2 e^{-3 / 2} e^{4 d_{2}} \sigma N^{1 / 2}\right)}{N}-\frac{4 d_{1}}{\sqrt{N}}+\frac{4 d_{3}}{N^{3 / 2}} .
$$

To obtain the final form of our scaling law, we introduce the surface area fraction of traps $f$, defined by $f=\pi N \sigma^{2} /(4 \pi)=N \sigma^{2} / 4$. Upon eliminating $N$ in (4.8), we obtain our desired scaling law for $C_{0}=C_{0}(f, \sigma)$,

$$
\frac{1}{C_{0}} \sim 1+\frac{\pi \sigma}{4 f}\left(1-\frac{8 d_{1}}{\pi} \sqrt{f}+\frac{\sigma}{\pi} \log (\beta \sqrt{f})+\frac{2 d_{3} \sigma^{2}}{\pi \sqrt{f}}\right)
$$

where $\beta \equiv 4 e^{-3 / 2} e^{4 d_{2}}$, which is asymptotically valid, provided that $f=\mathcal{O}\left(-\sigma^{2} \log \sigma\right)$. Finally, to derive our effective homogenized Robin boundary condition, we introduce $v_{h}$ satisfying

$$
\Delta v_{h}=0,|\mathbf{y}|>1 ; \quad \partial_{n} v_{h}+\kappa v_{h}=0,|\mathbf{y}|=1 ; \quad v_{h}(\mathbf{y}) \sim \frac{1}{|\mathbf{y}|}-\frac{1}{C_{h}} \quad \text { as }|\mathbf{y}| \rightarrow \infty,
$$

where we replace $C_{h}$ with $C_{0}$ in (4.9). The exact $v_{h}(\mathbf{y})=|\mathbf{y}|^{-1}-1-\kappa^{-1}$ yields $\kappa=C_{h} /\left(1-C_{h}\right)$, so that

$$
\kappa \sim \frac{4 f}{\pi \sigma}\left[1-\frac{8 d_{1}}{\pi} \sqrt{f}+\frac{\sigma}{\pi} \log (\beta \sqrt{f})+\frac{2 d_{3} \sigma^{2}}{\pi \sqrt{f}}\right]^{-1}, \quad \text { where } \beta \equiv 4 e^{-3 / 2} e^{4 d_{2}},
$$

provided that the nanotrap surface fraction satisfies $f=\mathcal{O}\left(-\sigma^{2} \log \sigma\right)$. In our main scaling law results (4.9) and (4.11), the coefficients $d_{i}$ for $i=1,2,3$, as defined in the energy expansion (4.6), can correspond either to those in the formal scaling law (4.4) for a uniform arrangement of points or to those from the true globally minimal energy for $\mathcal{H}$. We importantly remark, as discussed following (4.5) above, that the $8 d_{1} \sqrt{f} / \pi$ term in (4.11) is closely associated with internanotrap interactions for a uniform planar arrangement of nanotraps on a flat surface, while the other correction terms in (4.11) are intrinsically due to curvature effects of the sphere.

We now compare our scaling law for $\kappa$ in (4.11) with some other results in the literature $[4,3,34,51]$ derived from either physical considerations or parameter fittings. In dimensionless form, the Berg-Purcell result (cf. [4]) in (1.10) is

$$
\frac{1}{C_{0 \mathrm{bp}}}=1+\frac{\pi}{N \sigma}=1+\frac{\pi \sigma}{4 f}, \quad \kappa_{\mathrm{bp}}=\frac{N \sigma}{\pi}=\frac{4 f}{\pi \sigma},
$$

and so agrees with the leading terms in our results (4.9) and (4.11). We conclude that the Berg-Purcell result (1.10) holds in the small nanotrap surface fraction limit 
$f=\mathcal{O}\left(-\sigma^{2} \log \sigma\right) \ll 1$. In [51], the Berg-Purcell result (4.12) was modified based on physical considerations so as to explicitly account for the reduction of the surface area due to nanotraps, so that

$$
\kappa_{\mathrm{zw}}=\frac{4 f}{\pi \sigma(1-f)} .
$$

However, as seen by comparing (4.13) with (4.11), the small $f$ correction of $1 /(1-f) \approx$ $1+f+\cdots$ in (4.13) is subdominant to the more significant effect of intertrap interactions captured by the correction terms in (4.11). For a periodic arrangement of nanotraps on the surface of an infinite planar boundary, in $[3,34]$ the dimensionless leakage $\kappa$ for the corresponding half-space version of (1.5) was closely fitted to full numerical results by

$$
\kappa_{\mathrm{m}}=\frac{4 f}{\pi \sigma(1-f)^{2}}\left(1+A \sqrt{f}-B f^{2}\right)
$$

For a hexagonal array of nanotraps, the coefficients $A$ and $B$ were estimated in [2] from stochastic simulations as $A \approx 1.37$ and $B \approx 1.259$ in [3], and more accurately from a PDE-based finite-difference numerical method as $A \approx 1.49$ and $B \approx 0.92$ in [34]. To our knowledge there has been no such detailed parameter fittings of $\kappa$ for nanotraps on the sphere.

To compare (4.14) for planar nanotraps with our result (4.11) for nanotraps on the sphere, we let $f \ll 1$ in (4.14) and (4.11) and neglect the logarithmic terms in (4.11) due to the spherical surface. We get $\kappa_{\mathrm{m}} \approx 4 f /(\pi \sigma)(1+A \sqrt{f})$; however, using the binomial approximation, our result (4.11) yields $\kappa \approx 4 f /(\pi \sigma)\left(1+8 d_{1} \sqrt{f} / \pi\right)$. If we use the value $d_{1} \approx 0.55230$ for the optimal hexagonal-type pattern on the sphere, we calculate $8 d_{1} / \pi \approx 1.41$, which is remarkably close to the value $A$ predicted in [3] and [34] for (4.14). The minor difference in the predicted value is due to either the minor defects of the hexagonal pattern on the sphere or small numerical errors involved with either fitting the optimal discrete energy or the simulations in [3] and [34]. In any event, our expression (4.11) provides a "first principles" derivation of the effective leakage for the sphere, where the only parameters are associated with the large $N$ asymptotics of the discrete energy $\mathcal{H}$.

4.1. The effect of trap locations and trap fragmentation on the capacitance. In this section we investigate the effect of nanotrap fragmentation on the capacitance of the spherical target under the assumption that the total absorbing surface fraction $f$ is fixed and divided among $N$ identical nanotraps traps, of common radius $\sigma=2 \sqrt{f / N}$. To generate roughly equidistant points distributed on the sphere, we use the spiral Fibonacci points (cf. [44, 21, 49] and Appendix C) for different values of $N$ to generate the centers of the locally circular nanotraps. We avoid comparisons using random uniform nanotrap centers since configurations with a finite radius $\sigma$ will almost certainly generate overlapping patches for large enough $N$, thus violating the assumptions leading to (3.38).

Example 1 (fragmentation into $N$ equally spaced nanotraps). Here we consider $N$ identical nanotraps of common radius $\sigma=2 \sqrt{f / N}$ spaced equally on the surface of the entire sphere (see Figure 3(b)) for absorbing fractions $f=0.02,0.05,0.1,0.15$. In Figure 3(a) the capacitance $C_{0}^{-1}$ is seen to decrease monotonically as $N$ increases. The limiting value of $C_{0}^{-1}=1$ corresponds to an all-absorbing spherical target. We observe that fragmentation of the absorbing set greatly increases the capture rate. However, 


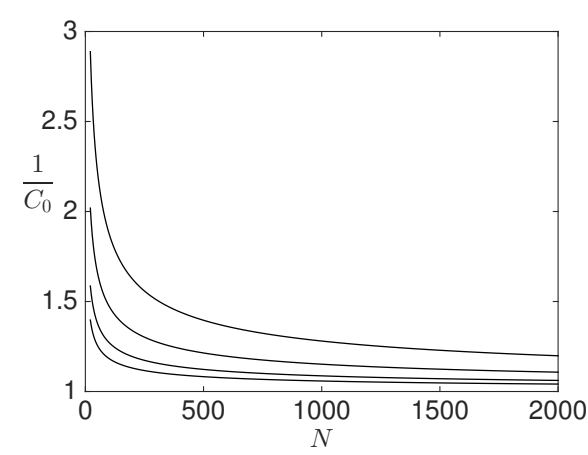

(a) Fragmentation of absorbing fraction into $N$ nanotraps.

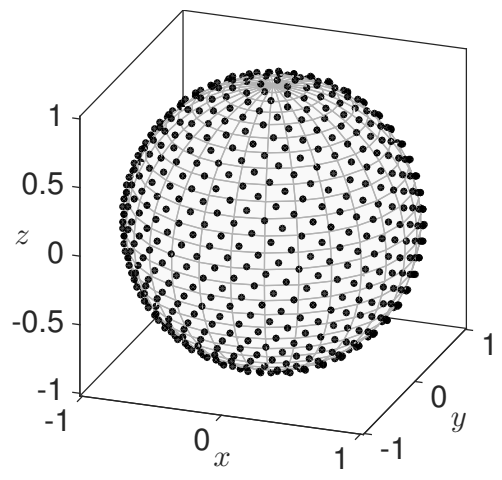

(b) $N=801$ nanotraps equally distributed on the unit sphere.

FIG. 3. (a) Fragmentation of the absorbing fraction into $N$ identical and equally spaced nanotraps. From top to bottom the curves correspond to the absorbing fractions $f=0.02,0.05,0.1,0.15$. As a confirmation of the homogenized formula for the numbers $N=2000, f=0.02$ (cf. [29, 41]), we calculate from (3.38) that $C_{0}^{-1}=1.1985$, which agrees well with $C_{h}^{-1}=1+\kappa^{-1} \approx 1.2028$ arising from the homogenized formula (4.11). (b) A sphere with an equispaced covering of $N=801$ points from a Fibonacci lattice (see Appendix C).

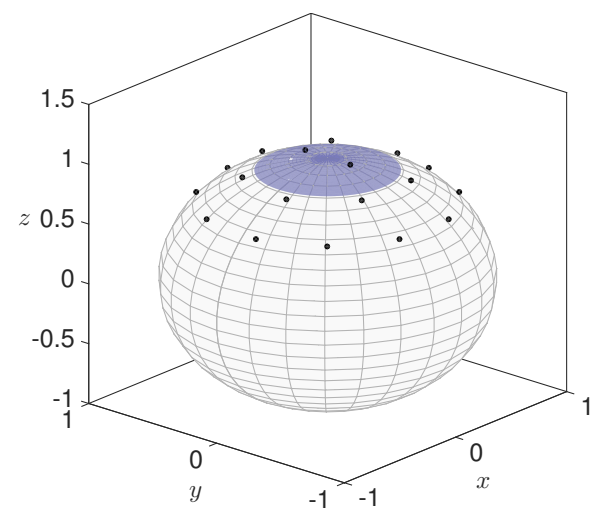

FIG. 4. $N=20$ identical and equally spaced nanotraps (centers shown only) in the polar region $\theta \in\left(0, \frac{\pi}{3}\right)$ with total cumulative absorbing fraction $f=0.05$. The shaded region represents a single nanotrap whose area is equivalent to the 20 smaller nanotraps.

once $N$ is already large, there is only a very marginal increase in $C_{0}$ obtained from increasing $N$ further.

Example 2 (clustering near the north pole). Here we compare the capacitance of a single nanotrap after fragmentation into $N=20$ identical nanotraps of common radius $\sigma=2 \sqrt{f / N}$ for $f=0.05$. The nanotraps are equally spaced and clustered in the polar region of the sphere $\theta \in(0, \pi / 3)$. In Figure 4 the shaded region represents the absorbing area as a single nanotrap. Using formula (3.38), we calculate that

$$
\left.\frac{1}{C_{0}}=5.413 \quad \text { (single trap }\right), \quad \frac{1}{C_{0}}=2.788 \quad(20 \text { fragmented nanotraps }) .
$$

Hence the subdividing of a single nanotrap into 20 smaller nanotraps of equivalent cumulative area roughly halves the capacitance and, consequently, the mean capture 
TABLE 1

Comparison of leakage parameter in Robin condition as predicted by full discrete energy (second column) $\kappa_{0}=\left[-1+1 / C_{0}\right]^{-1}$, where $C_{0}$ is computed from (3.38a), the scaling law $\kappa$ of (4.11) (third column), and the truncated scaling law (last column) $\kappa_{t} \sim \frac{4 f}{\pi \sigma}\left[1-\frac{8 d_{1}}{\pi} \sqrt{f}\right]^{-1}$ with $d_{1}=0.5$. The nanotrap coverage is $f=0.02$, and the nanotrap radius $\sigma$ is $\sigma=2 \sqrt{f / N}$. The centers of the nanotraps are given by the spiral Fibonacci points (see Appendix C).

\begin{tabular}{|c|c|c|c|}
\hline$N$ & $\kappa_{0}$ & $\kappa$ & $\kappa_{\mathrm{t}}$ \\
\hline 10 & 0.36817 & 0.36303 & 0.34723 \\
20 & 0.50909 & 0.50784 & 0.49105 \\
40 & 0.71202 & 0.71190 & 0.69446 \\
80 & 1.0108 & 1.0000 & 0.98211 \\
160 & 1.4275 & 1.4071 & 1.3889 \\
\hline
\end{tabular}

time to the spherical target from (1.4).

Example 3 (effects of curvature on homogenized scaling law). Here we compare our scaling law for $\kappa$ in (4.11), in which we use the values $d_{1}=1 / 2, d_{2}=1 / 8$, and $d_{3}=1 / 4$, with the corresponding result $\kappa_{0}=\left[-1+1 / C_{0}\right]^{-1}$ obtained by computing $1 / C_{0}$ from the discrete energy in (3.38a) for a given configuration of centers of the nanotraps. In our comparison, we show the results obtained when using the truncated scaling law $\kappa_{\mathrm{t}} \sim \frac{4 f}{\pi \sigma}\left[1-\frac{8 d_{1}}{\pi} \sqrt{f}\right]^{-1}$, which neglects the effect of the curvature of the sphere. The results are shown in Table 1 for different $N$ while maintaining $f=0.02$, i.e., a $2 \%$ nanotrap coverage of the sphere. We observe that even at $N=10,20$, our scaling law accurately predicts $\kappa_{0}$, and that the effect of curvature at smaller values of $N$ is more important than at higher values of $N$, as expected.

5. Numerical solutions and validation. In this section we validate the asymptotic formulas (3.38) and (3.40) by comparing to results from a spectral boundary element solution of the exterior mixed Neumann-Dirichlet boundary value problem (1.5). The numerical problem is formulated as a linear integral equation, specifically a Neumann-to-Dirichlet map on the set of nanotraps, $\Gamma_{a}$. This approach relates the known surface potential, $\left.u\right|_{\partial \Omega_{0}}=p(\mathbf{y})$, to the surface flux, $\left.\partial_{n} u\right|_{\partial \Omega_{0}}=-\left.\partial_{r} u\right|_{\partial \Omega_{0}}=$ $q(\mathbf{y})$, which is unknown on $\Gamma_{a}$ and vanishes on $\Gamma_{r}$. The exact solution to the Neumann problem is known in terms of the surface Green's function (1.7),

$$
u(\mathbf{y})=\int_{\mathbf{x} \in \Omega_{0}} G(\mathbf{y} ; \mathbf{x}) q(\mathbf{x}) d S, \quad \mathbf{y} \in \mathbb{R}^{3} \backslash \Omega_{0} .
$$

This formulation simplifies considerably by noting that the surface flux, $q(\mathbf{x})$, is nonzero only on the nanotraps, $\Gamma_{a}$, and by restricting our interest to the surface of the sphere where $u(\mathbf{y})=p(\mathbf{y})$. This yields the linear integral equation

$$
p(\mathbf{y})=\mathcal{A}[q(\mathbf{x})] \equiv \int_{\mathbf{x} \in \Gamma_{a}} g(|\mathbf{x}-\mathbf{y}|) q(\mathbf{x}) d S, \quad \mathbf{y} \in \Omega_{0},
$$

where the kernel of the integral operator is defined by the Green's function restricted to the sphere,

$$
G(\mathbf{y} ; \mathbf{x})=\frac{1}{2 \pi} g(|\mathbf{x}-\mathbf{y}|) \quad \text { for } \quad \mathbf{x}, \mathbf{y} \in \Omega_{0}, \quad g(\mu) \equiv \frac{1}{\mu}+\frac{1}{2} \log \left(\frac{\mu}{2+\mu}\right),
$$

as defined in (1.8b). Equation (5.1) is solved pseudospectrally with a judicious choice of basis of functions for the surface potential, $p(\mathbf{y})$, and the surface flux, $q(\mathbf{y})$, within 
the nanotraps $\Gamma_{a}$. This choice, based on Zernike polynomials [36], captures properties of the exact solution for a single absorbing circular trap on a half plane (3.11) (cf. [19]), particularly singularities in the flux along $\partial \Gamma_{a}$, a notorious feature of mixed NeumannDirichlet boundary value problems $[48,17]$.

The method is effective and accurate for small, widely separated nanotraps, which is the relevant asymptotic and biological limit. Accuracy is degraded if trap boundaries are nearly touching. Further details of this method, refinement studies, and quantification of the errors will be described in a companion paper [6]. Below we focus on numerically validating the single and multiple nanotrap asymptotic formulae derived previously.

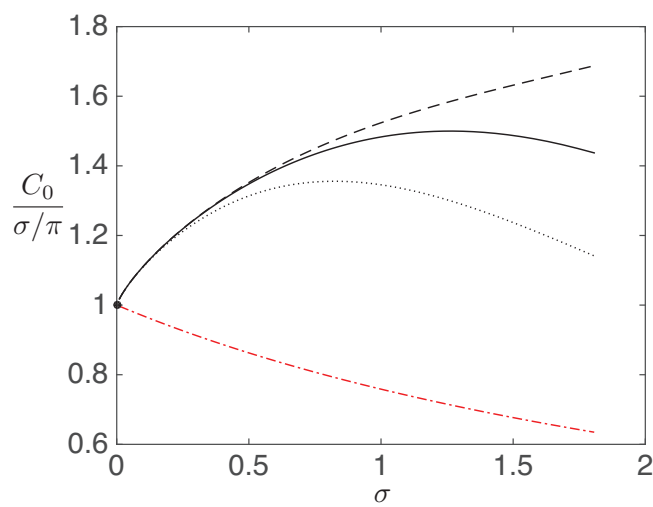

(a) Rescaled capacitance $C_{0} /(\sigma / \pi)$ against trap radius $\sigma$.

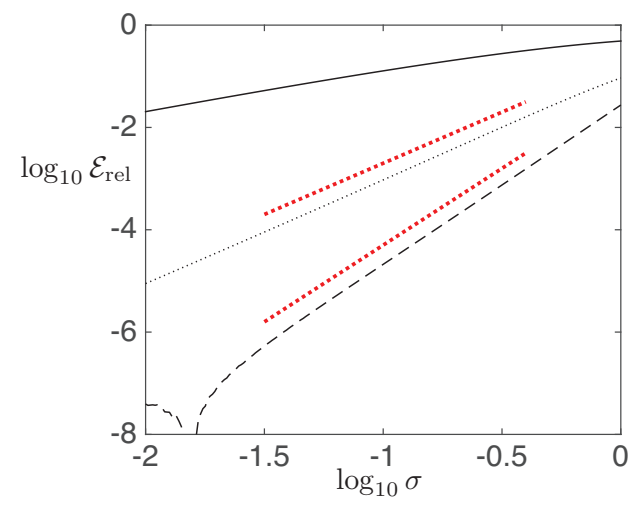

(b) Relative errors $\mathcal{E}_{\text {rel }}$ on logarithmic scale.

FIG. 5. Comparison of asymptotic predictions and full numerics for a single trap of radius $\sigma$. (a) Three-term (dotted line) expansion (3.39), four-term (dashed line) expansion (3.40), full numerics (solid line), and Burg-Purcell approximation (4.12) (dot-dashed line). (b) Relative errors of asymptotic approximations for the capacitance as $\sigma \rightarrow 0$. Solid line is the leading order term with three-term (thin dotted line) and four-term (dashed line) expansions. The two thick dotted lines are of slope 2 (upper) and 3 (lower) indicating the order of the error in the expansion. Numerical error is seen to arise as the relative error approaches $10^{-8}$.

5.1. Single nanotrap. For the single trap case, we verify the accuracy of the single trap asymptotics (3.39) obtained from the matched asymptotic approach and also from the exact approach (3.40) as $\sigma \rightarrow 0$. The results for the rescaled capacitance in the single patch case are shown in Figure 5(a) and demonstrate that the asymptotic formulae (3.39) and (3.40) are quite accurate, even when the trap radius becomes $\mathcal{O}(1)$. The dot-dashed curve in Figure 5(a) indicates the classic Berg-Purcell approximation (4.12), which is significantly less accurate for $N=1$. In Figure $5(\mathrm{~b})$, the numerical results give validation of the relative errors of the asymptotic formula as $\sigma \rightarrow 0$ and reveal that round-off error limits the smallest relative error obtainable to about one part in $10^{8}$.

5.2. Multiple nanotraps. Here we compare the asymptotic approximation for multiple nanotraps of common radius (3.38) against full numerical calculation of the rescaled capacitance. The validity of (3.38) is demonstrated for nanotraps centered at the vertices of the regular Platonic solids and also Fibonacci spiral points for $N \leq 20$. We note that the vertices of the regular Platonic solids have many symmetries which can potentially obscure errors in the numerical procedure. The Fibonacci spiral points 


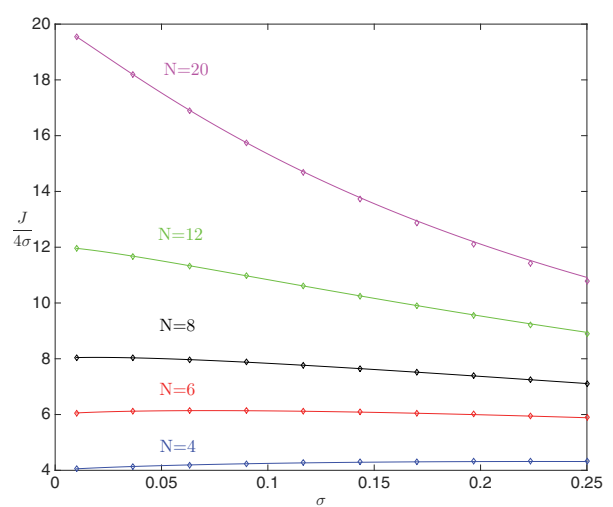

(a) Comparison with regular Platonic points.

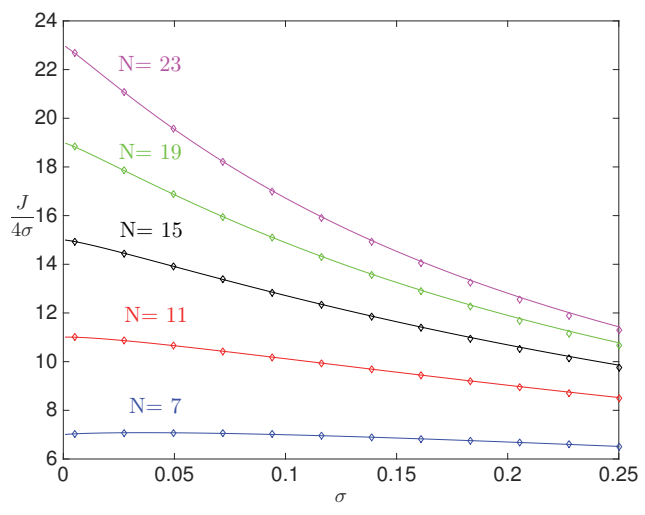

(b) Comparison with Fibonacci lattice points.

FIG. 6. Comparison of the rescaled flux $J /(4 \sigma)$ as predicted by the asymptotic formula (solid lines) and full numerics (diamonds) with nanotrap locations given by vertices of the regular Platonic solids (panel (a)) and Fibonacci spirals (panel (b)). The Fibonacci spiral algorithm (Appendix C) generates an odd number of equispaced points on the sphere.

(Appendix C) do not share these symmetries and therefore provide a good benchmark for evaluation of the numerical method.

In both cases, Figure 6 shows excellent agreement for configurations up to $N=21$ nanotraps. The rescaled flux $J /(4 \sigma)$, where $J=4 \pi C_{0}$, has the property that

$$
\lim _{\sigma \rightarrow 0} \frac{J}{4 \sigma}=\lim _{\sigma \rightarrow 0} \frac{C_{0}}{(\sigma / \pi)}=N,
$$

in agreement with (3.38) and the original Berg-Purcell result (4.12), and as observed in each curve of Figure 6.

6. Asymptotics of an exact solution for a single boundary trap. In this section we derive the result (3.40) for the capacitance of a spherical target of unit radius that has a single locally circular nanotrap of radius $\sigma \ll 1$ and azimuthal extent $\alpha$. This is accomplished by solving (1.5) exactly in the form of a dual Legendre series. We center the nanotrap at the north pole, so that with azimuthal symmetry (1.5) reduces to determining $v(r, \theta)$ that satisfies

$$
\begin{gathered}
\Delta v \equiv \frac{\partial^{2} v}{\partial r^{2}}+\frac{2}{r} \frac{\partial v}{\partial r}+\frac{\cot \theta}{r^{2}} \frac{\partial v}{\partial \theta}+\frac{1}{r^{2}} \frac{\partial^{2} v}{\partial \theta^{2}}=0, \quad r>1, \quad 0 \leq \theta \leq \pi, \\
v=0 \quad \text { on } \quad r=1, \quad 0 \leq \theta \leq \alpha ; \quad v_{r}=0 \quad \text { on } \quad r=1, \quad \alpha<\theta \leq \pi, \\
v \sim \frac{1}{r}+A_{0} \quad \text { as } \quad r \rightarrow \infty .
\end{gathered}
$$

Our goal is to calculate the constant $A_{0}$ from this problem, which determines the capacitance as $C_{0}=-1 / A_{0}$. When comparing to the asymptotic formula (3.39), it is important to note that $\alpha$ measures the azimuthal extent of the nanotrap, whereas $\sigma$ measures its radius. Therefore, when comparing (3.39) to the expression for $C_{0}$ obtained from (6.1), we use the relationship as $\alpha \rightarrow 0$ and $\sigma \rightarrow 0$,

$$
\sigma=2 \sin \left(\frac{\alpha}{2}\right) \quad \Longrightarrow \quad \alpha=\sigma+\frac{\sigma^{3}}{24}+\mathcal{O}\left(\sigma^{5}\right) .
$$


The series solution of (6.1) has a Legendre expansion of the form

$$
v(r, \theta)=\frac{1}{r}+A_{0}+\sum_{n=1}^{\infty} A_{n} r^{-(n+1)} P_{n}[\cos \theta]
$$

By applying the boundary conditions we obtain a dual Legendre series for the unknown coefficients $A_{n}$, written as

$$
\sum_{n=0}^{\infty} A_{n} P_{n}[\cos \theta]=-1, \quad 0 \leq \theta \leq \alpha ; \quad \sum_{n=1}^{\infty}(n+1) A_{n} P_{n}[\cos \theta]=-1, \quad \alpha<\theta \leq \pi
$$

To solve this dual series problem, we follow the methodology of (cf. [47, 14]). We first rescale the coefficients using

$$
A_{0}=b_{0} ; \quad A_{n}=\left[1-\frac{1}{2(n+1)}\right] b_{n}, \quad n \geq 1
$$

so as to obtain the related dual series

$$
\left\{\begin{array}{cl}
\sum_{n=0}^{\infty}\left(1+M_{n}\right) b_{n} P_{n}[\cos \theta]=f_{0} \equiv-1, \quad & 0<\theta \leq \alpha, \\
\sum_{n=0}^{\infty}(2 n+1) b_{n} P_{n}[\cos \theta]=g_{0} \equiv-2+b_{0}, & \alpha<\theta \leq \pi,
\end{array} \quad M_{n}=\left\{\begin{array}{cc}
0, & n=0 \\
\frac{-1}{2(n+1)}, & n \geq 1 .
\end{array}\right.\right.
$$

There are three key results and identities that are needed in order to obtain the solution for the coefficients $b_{n}$. The first is the solution of the following integral equation:

$$
\int_{0}^{\theta} \frac{p(u) d u}{\sqrt{\cos u-\cos \theta}}=q(\theta) \quad \Longleftrightarrow \quad p(u)=\frac{1}{\pi} \frac{d}{d u} \int_{0}^{u} \frac{q(\theta) \sin \theta d \theta}{\sqrt{\cos \theta-\cos u}}
$$

The two remaining identities required in the analysis are

$P_{n}[\cos \theta]=\frac{\sqrt{2}}{\pi} \int_{0}^{\theta} \frac{\cos \left(n+\frac{1}{2}\right) u d u}{\sqrt{\cos u-\cos \theta}}, \quad \sqrt{2} \sum_{n=0}^{\infty} P_{n}[\cos \alpha] \cos \left(n+\frac{1}{2}\right) u=\frac{H(\alpha-u)}{\sqrt{\cos u-\cos \theta}}$,

where $H(z)$ is the Heaviside function. The first step is to formulate a Legendre expansion for the solution on the Dirichlet portion of the domain:

$$
h(\theta)=\sum_{n=0}^{\infty}(2 n+1) b_{n} P_{n}[\cos \theta], \quad 0 \leq \theta \leq \alpha .
$$

Then, by inverting this expansion, we obtain that

$$
b_{n}=\frac{1}{2} \int_{0}^{\alpha} h(\theta) P_{n}[\cos \theta] \sin \theta d \theta+\frac{1}{2} \int_{\alpha}^{\pi} g_{0} P_{n}[\cos \theta] \sin \theta d \theta .
$$


Substituting this expression for $b_{n}$ into the first equation of (6.6) gives the equation

$$
\begin{aligned}
f_{0}= & \frac{1}{2} \int_{0}^{\alpha} h(s) \sin s\left[\sum_{n=0}^{\infty} P_{n}[\cos s] P_{n}[\cos \theta]\right] d s+\frac{1}{2} \int_{\alpha}^{\pi} g_{0} \sin s\left[\sum_{n=0}^{\infty} P_{n}[\cos s] P_{n}[\cos \theta]\right] d s \\
& +\frac{1}{2} \int_{0}^{\alpha} h(s) \sin s\left[\sum_{n=0}^{\infty} M_{n} P_{n}[\cos s] P_{n}[\cos \theta]\right] d s \\
& +\frac{1}{2} \int_{\alpha}^{\pi} g_{0} \sin s\left[\sum_{n=0}^{\infty} M_{n} P_{n}[\cos s] P_{n}[\cos \theta]\right] d s \\
= & \mathcal{I}_{1}+\mathcal{I}_{2}+\mathcal{I}_{3}+\mathcal{I}_{4} .
\end{aligned}
$$

The identities (6.8) can be applied to sum the series

$$
\begin{aligned}
\sum_{n=0}^{\infty} P_{n}[\cos s] P_{n}[\cos \theta] & =\frac{\sqrt{2}}{\pi} \int_{0}^{\theta} \sum_{n=0}^{\infty} \frac{P_{n}[\cos s] \cos \left(n+\frac{1}{2}\right) u}{\sqrt{\cos u-\cos \theta}} d u \\
& =\frac{1}{\pi} \int_{0}^{\theta} \frac{H(s-u) d u}{\sqrt{\cos u-\cos \theta} \sqrt{\cos u-\cos s}}
\end{aligned}
$$

and so, after exchanging the order of integration, $\mathcal{I}_{1}$ becomes

$$
\mathcal{I}_{1}=\frac{1}{2 \pi} \int_{0}^{\alpha} h(s) \sin s \int_{0}^{\theta} \frac{H(s-u) d u}{\sqrt{\cos u-\cos \theta} \sqrt{\cos u-\cos s}}=\int_{0}^{\alpha} \frac{\hat{H}(u) d u}{\sqrt{\cos u-\cos \theta}},
$$

where $\hat{H}(u)$ satisfies

$$
\hat{H}(u)=\frac{1}{2 \pi} \int_{u}^{\alpha} \frac{h(s) \sin s d s}{\sqrt{\cos u-\cos s}} \quad \Longleftrightarrow \quad h(\theta)=\frac{-2}{\sin \theta} \frac{d}{d \theta} \int_{\theta}^{\alpha} \frac{\hat{H}(u) \sin u d u}{\sqrt{\cos \theta-\cos u}} .
$$

Similar calculations for $\mathcal{I}_{2}$ yield that

$$
\mathcal{I}_{2}=\int_{0}^{\theta} \frac{\hat{G}(u, \alpha) d u}{\sqrt{\cos u-\cos \theta}}, \quad \text { where } \quad \hat{G}(u, \alpha)=\frac{1}{2 \pi} \int_{\alpha}^{\pi} \frac{g_{0} \sin s d s}{\sqrt{\cos u-\cos s}} .
$$

In the calculations for $\mathcal{I}_{3}$, we again use the first identity in (6.8) to obtain that

$$
\begin{aligned}
\mathcal{I}_{3} & =\frac{1}{2} \sum_{n=0}^{\infty} M_{n} \int_{0}^{\alpha} h(s) \sin s P_{n}[\cos \theta] P_{n}[\cos s] d s \\
& =\frac{1}{\pi^{2}} \sum_{n=0}^{\infty} M_{n} \int_{0}^{\alpha} h(s) \sin s d s \int_{0}^{\theta} \frac{\cos \left(n+\frac{1}{2}\right) u d u}{\sqrt{\cos u-\cos \theta}} \int_{0}^{s} \frac{\cos \left(n+\frac{1}{2}\right) v d v}{\sqrt{\cos v-\cos s}} \\
& =\int_{0}^{\theta} \frac{d u}{\sqrt{\cos u-\cos \theta}} \int_{0}^{\alpha} \hat{H}(v) K(u, v) d v .
\end{aligned}
$$

In the final step above, the order of integration was switched and the function $K(u, v)$ was defined as

$$
K(u, v)=\frac{2}{\pi} \sum_{n=0}^{\infty} M_{n} \cos \left(n+\frac{1}{2}\right) u \cos \left(n+\frac{1}{2}\right) v .
$$


This series defining $K(u, v)$ can be summed using the terms $M_{n}$ in (6.6) to obtain that

$$
K(u, v)=\frac{1}{4 \pi}\left[\begin{array}{c}
2\left(1+\log 2 \sin \frac{u+v}{2}\right) \cos \frac{u+v}{2}+(u+v-\pi) \sin \frac{u+v}{2} \\
+2\left(1+\log 2 \sin \frac{u-v}{2}\right) \cos \frac{u-v}{2}+(u-v-\pi) \sin \frac{u-v}{2}
\end{array}\right] .
$$

The final integral $\mathcal{I}_{4}$ is similarly calculated to be

$$
\mathcal{I}_{4}=\int_{0}^{\theta} \frac{d u}{\sqrt{\cos u-\cos \theta}}\left[\int_{0}^{\alpha} \hat{G}(v, \alpha) K(u, v) d v+\int_{\alpha}^{\pi} \hat{G}(v, v) K(u, v) d v\right] .
$$

The equation $\mathcal{I}_{1}+\mathcal{I}_{2}+\mathcal{I}_{3}+\mathcal{I}_{4}=f_{0}$ is an integral equation of type (6.7). Therefore, the solution satisfies

$$
J(u)+\int_{0}^{\alpha} J(v) K(u, v) d v=M(u)
$$

where

$J(u)=\hat{H}(u)+\hat{G}(u, \alpha), \quad M(u)=\frac{1}{\pi} \frac{d}{d u} \int_{0}^{u} \frac{f_{0} \sin \theta d \theta}{\sqrt{\cos \theta-\cos u}}-\int_{\alpha}^{\pi} \hat{G}(v, v) K(u, v) d v$.

The expression for $b_{0}$ is now obtained under the assumption that $f_{0}$ and $g_{0}$ are constants. Starting from (6.10), we have

$$
b_{0}=\frac{1}{2} \int_{0}^{\alpha} h(\theta) \sin \theta d \theta+\frac{1}{2} \int_{\alpha}^{\pi} g_{0} \sin \theta d \theta=\frac{1}{2} \int_{0}^{\alpha} h(\theta) \sin \theta d \theta+g_{0} \cos ^{2}\left(\frac{\alpha}{2}\right) .
$$

Then, by using $(6.13)$ and $\hat{H}(u)=J(u)-\hat{G}(u, \alpha)$, we obtain that

$$
b_{0}=\sqrt{2} \int_{0}^{\alpha} J(u) \cos \left(\frac{u}{2}\right) d u-\sqrt{2} \int_{0}^{\alpha} \hat{G}(u, \alpha) \cos \left(\frac{u}{2}\right) d u+g_{0} \cos ^{2}\left(\frac{\alpha}{2}\right) .
$$

Next, from (6.13), we have

$$
\hat{G}(u, \alpha)=\frac{1}{2 \pi} \int_{\alpha}^{\pi} \frac{g_{0} \sin s d s}{\sqrt{\cos u-\cos s}}=\frac{g_{0}}{\pi}\left[\sqrt{2} \cos \left(\frac{u}{2}\right)-\sqrt{\cos u-\cos \alpha}\right],
$$

so that

$$
\sqrt{2} \int_{0}^{\alpha} \hat{G}(u, \alpha) \cos \left(\frac{u}{2}\right) d u=\frac{g_{0}}{\pi}\left[\alpha+\sin \alpha-\pi \sin ^{2}\left(\frac{\alpha}{2}\right)\right] .
$$

Upon substituting (6.21) into (6.20), we get

$$
b_{0}=\sqrt{2} \int_{0}^{\alpha} J(u) \cos \left(\frac{u}{2}\right) d u-\frac{g_{0}}{\pi}(\alpha+\sin \alpha-\pi),
$$

where $J(u)$ satisfies the integral equation (6.18). At this stage, we seek an asymptotic approximation for $\int_{0}^{\alpha} J(u) \cos \left(\frac{u}{2}\right) d u$ in the limit $\alpha \rightarrow 0$. From (6.18), we have that

$$
\begin{aligned}
& \sqrt{2} \int_{0}^{\alpha} J(u) \cos \left(\frac{u}{2}\right) d u+\sqrt{2} \int_{0}^{\alpha} \int_{0}^{\alpha} J(v) K(u, v) \cos \left(\frac{u}{2}\right) d v d u \\
= & \sqrt{2} \int_{0}^{\alpha} M(u) \cos \left(\frac{u}{2}\right) d u
\end{aligned}
$$


where we can simplify $M(u)$ further using (6.21) to give

$$
M(u)=\frac{\sqrt{2} f_{0}}{\pi} \cos \left(\frac{u}{2}\right)+\frac{\sqrt{2} g_{0}}{\pi} \int_{0}^{\alpha} \cos \left(\frac{v}{2}\right) K(u, v) d v .
$$

In the final term on the right-hand side of (6.23), the orthogonality condition $\int_{0}^{\pi} K(u, v) \cos \left(\frac{u}{2}\right) d u=0$ implies $\int_{\alpha}^{\pi} K(u, v) \cos \left(\frac{u}{2}\right) d u=-\int_{0}^{\alpha} K(u, v) \cos \left(\frac{u}{2}\right) d u$. The right-hand side of (6.22) simplifies to

$$
\begin{aligned}
\sqrt{2} \int_{0}^{\alpha} M(u) \cos \left(\frac{u}{2}\right) d u & =\frac{f_{0}}{\pi}(\alpha+\sin \alpha)+\frac{2 g_{0}}{\pi} \int_{0}^{\alpha} \int_{0}^{\alpha} K(u, v) \cos \left(\frac{u}{2}\right) \cos \left(\frac{v}{2}\right) d u d v \\
& =\frac{f_{0}}{\pi}(\alpha+\sin \alpha)+\frac{2 g_{0}}{\pi} \mathcal{I}_{K_{1}} .
\end{aligned}
$$

By replacing $K(u, v)$, given in (6.16b), by its small argument approximation, the integral term $\mathcal{I}_{K_{1}}$ on the right-hand side of (6.24) is found to have the behavior

$$
\begin{aligned}
\mathcal{I}_{K_{1}} & =\int_{0}^{\alpha} \int_{0}^{\alpha} K(u, v) \cos \left(\frac{u}{2}\right) \cos \left(\frac{v}{2}\right) d u d v \\
& =\frac{\alpha^{2}}{2 \pi}[-1+2 \log \alpha+\log 4]-\frac{\alpha^{3}}{8}+\mathcal{O}\left(\alpha^{4} \log \alpha\right) \text { as } \alpha \rightarrow 0 .
\end{aligned}
$$

The remaining term $\int_{0}^{\alpha} \int_{0}^{\alpha} J(v) K(u, v) \cos \left(\frac{u}{2}\right) d v d u$ in (6.22) is now estimated for $\alpha \ll 1$. Following from [47, sections 3.2.3-3.2.6] and applying the Cauchy-Schwarz inequality to the integral equation (6.18), we obtain that

$$
|J(u)-M(u)|=\left|\int_{0}^{\alpha} K(u, v) J(u) d v\right| \leq\|K\|_{2}\|J\|_{2} \ll 1 \quad \text { as } \quad \alpha \rightarrow 0,
$$

where $\|M\|_{2}=\sqrt{\int_{0}^{\alpha} M(u)^{2} d u}$. The logarithmic singularity of $K(u, v)$ and (6.23) means that (cf. Appendix A of [47])

$$
\begin{gathered}
\|M\|_{2}=\mathcal{O}(\sqrt{\alpha}), \quad\|J\|_{2}=\mathcal{O}(\sqrt{\alpha}), \quad\|K\|_{2}=\mathcal{O}(\alpha \log \alpha), \quad \alpha \rightarrow 0 . \\
J(u)=M(u)+\mathcal{O}\left(\|K\|_{2}\|M\|_{2}\right)=M(u)+\mathcal{O}\left(\alpha^{3 / 2} \log \alpha\right)
\end{gathered}
$$

Therefore, we may now estimate the second integral of (6.22) using (6.26b) and (6.23) to obtain

$$
\begin{aligned}
& \sqrt{2} \int_{0}^{\alpha} \int_{0}^{\alpha} J(v) K(u, v) \cos \left(\frac{u}{2}\right) d v d u \\
& =\sqrt{2} \int_{0}^{\alpha} \int_{0}^{\alpha}\left[M(v)+\mathcal{O}\left(\alpha^{3 / 2} \log \alpha\right)\right] K(u, v) \cos \left(\frac{u}{2}\right) d v d u \\
& =\int_{0}^{\alpha} \int_{0}^{\alpha}\left[\frac{2 f_{0}}{\pi} \cos \left(\frac{v}{2}\right)+\frac{2 g_{0}}{\pi} \int_{0}^{\alpha} \cos \left(\frac{s}{2}\right) K(v, s) d s\right] K(u, v) \cos \left(\frac{u}{2}\right) d v d u \\
& =\frac{2 f_{0}}{\pi} \mathcal{I}_{K_{1}}+\frac{2 g_{0}}{\pi} \mathcal{I}_{K_{2}}+\mathcal{O}\left(\alpha^{7 / 2} \log ^{2} \alpha\right)
\end{aligned}
$$


where $\mathcal{I}_{K_{1}}$ was given in $(6.25)$ and $\mathcal{I}_{K_{2}}$ satisfies

$$
\begin{aligned}
\mathcal{I}_{K_{2}} & =\int_{0}^{\alpha} \int_{0}^{\alpha}\left[\int_{0}^{\alpha} \cos \left(\frac{s}{2}\right) K(v, s) d s\right] K(u, v) \cos \left(\frac{u}{2}\right) d v d u \\
& =\frac{\alpha^{3}}{\pi^{2}}\left[\log ^{2} \alpha+(2 \log 2-1) \log \alpha+\log ^{2} 2-\log 2+\frac{15-\pi^{2}}{18}\right] \quad \text { as } \quad \alpha \rightarrow 0 .
\end{aligned}
$$

Therefore, (6.24) together with (6.25) and (6.27) yields that

$$
\begin{aligned}
& \sqrt{2} \int_{0}^{\alpha} J(u) \cos \left(\frac{u}{2}\right) d u \\
= & \sqrt{2} \int_{0}^{\alpha} M(u) \cos \left(\frac{u}{2}\right) d u-\sqrt{2} \int_{0}^{\alpha} \int_{0}^{\alpha} J(v) K(u, v) \cos \left(\frac{u}{2}\right) d v d u \\
= & \frac{f_{0}}{\pi}\left(2 \alpha-\frac{\alpha^{3}}{6}\right)+\frac{2 g_{0}}{\pi} \mathcal{I}_{K_{1}}-\frac{2 f_{0}}{\pi} \mathcal{I}_{K_{1}}-\frac{2 g_{0}}{\pi} \mathcal{I}_{K_{2}}+\mathcal{O}\left(\alpha^{7 / 2} \log ^{2} \alpha\right) \quad \text { as } \quad \alpha \rightarrow 0 .
\end{aligned}
$$

The final expression for $b_{0}$ arises from substituting (6.29) into (6.21) to yield that

$b_{0}=\frac{f_{0}}{\pi}\left(2 \alpha-\frac{\alpha^{3}}{6}\right)+\frac{2 g_{0}}{\pi} \mathcal{I}_{K_{1}}-\frac{2 f_{0}}{\pi} \mathcal{I}_{K_{1}}-\frac{2 g_{0}}{\pi} \mathcal{I}_{K_{2}}-\frac{g_{0}}{\pi}\left(2 \alpha-\frac{\alpha^{3}}{6}-\pi\right) \quad$ as $\alpha \rightarrow 0$.

Applying the values $f_{0}=-1$ and $g_{0}=-2+b_{0}$ and solving the resulting algebraic equation for $b_{0}$, we conclude that

$b_{0}=-\frac{\pi}{\alpha}\left[1+\frac{\alpha}{\pi}\left(\log \alpha-\frac{3}{2}+\log 2\right)+\frac{\alpha^{2}}{\pi^{2}}\left(\frac{\pi^{2}-42}{72}\right)+\mathcal{O}\left(\alpha^{3} \log ^{3} \alpha\right)\right] \quad$ as $\alpha \rightarrow 0$.

Returning to an expression in terms of the nanotrap radius $\sigma$ using (6.2) and $\alpha \sim$ $\sigma+\sigma^{3} / 24$ as $\sigma \rightarrow 0$, while also recalling from (6.5) that $b_{0}=A_{0}$ and that $A_{0}=-1 / C_{0}$, where $C_{0}$ is the capacitance of the spherical target, we conclude that the right-hand side of (6.30) agrees with the expression for $-1 / C_{0}$ as derived in (3.39) from the matched asymptotic expansion approach, with an improved error estimate. The result for the capacitance is

(6.31)

$\frac{1}{C_{0}}=\frac{\pi}{\sigma}\left[1+\frac{\sigma}{\pi}\left(\log \sigma-\frac{3}{2}+\log 2\right)-\frac{\sigma^{2}}{\pi^{2}}\left(\frac{\pi^{2}+21}{36}\right)+\mathcal{O}\left(\sigma^{3} \log \sigma\right)\right] \quad$ as $\quad \sigma \rightarrow 0$

The absence of $\mathcal{O}\left(\sigma^{2} \log ^{2} \sigma, \sigma^{2} \log \sigma\right)$ terms in (6.31) arises from cancellations which are particular to the $N=1$ case, as also seen with the $\mathcal{O}\left(\sigma^{2} \log ^{2} \sigma\right)$ term of (3.37). In Figure 5, a favorable comparison of (6.31) with full numerical simulations is shown.

7. Discussion. We have analyzed the MFPT and standard deviation for Brownian motion in a bounded 3-D domain that contains a small structured spherical target of radius $\varepsilon \ll 1$ whose boundary has a collection of even smaller disk-shaped surface nanotraps of radii $\mathcal{O}(\varepsilon \sigma)$ with $\sigma \ll 1$ on an otherwise reflecting surface. The MFPT and the standard deviation both depend on the capacitance $C_{0}$ associated with the target. We have established the new result that the average MFPT, pertaining to a 
uniform distribution of initial points for the Brownian walk, and the standard deviation are identical up to two orders in $\varepsilon$. As a result, the MFPT may not in itself be a reliable predictor of the capture time for a pure Brownian walk. It would be interesting to extend this analysis to compare the MFPT and standard deviation for a narrow capture process where there is a biased random walk or drift directed to the target site (cf. $[28,29])$.

Our main focus was the derivation and numerical validation of an asymptotic expansion for the capacitance $C_{0}$ of the structured target in the limit $\sigma \ll 1$ of small nanotrap radius for a finite collection of $N$ circular nanotraps. We have shown that $C_{0}$ depends significantly on the spatial configuration of nanotraps and the fragmentation of the nanotrap set. The capacitance $C_{0}$ was shown to be maximized, and the corresponding MFPT minimized, when the configuration $\left\{\mathbf{y}_{1}, \ldots, \mathbf{y}_{N}\right\}$ of the centers of the nanotraps minimize a certain discrete energy $\mathcal{H}$ related to pairwise interactions induced by the surface Neumann Green's function. This discrete energy is a generalization of the classical Fekete point interaction energy (cf. [44]) and is similar to that derived for the narrow escape problem in [12]. It would be interesting to use the computational optimization framework of [12] to numerically identify optimal configurations of $N$ nanotraps of a common radius that maximize $C_{0}$. It would also be worthwhile to provide a rigorous proof of our formula for $C_{0}$ by extending the approach of [10] used to prove a related result of [12] for the narrow escape problem.

In the limit $N \rightarrow \infty$, with scaling $N \leq \mathcal{O}(-\log \sigma)$, we have used the large $N$ asymptotics of the discrete energy for a spatially uniform configuration of nanotraps to derive a new homogenized expression for $C_{0}$ in the low nanotrap surface area fraction $f=\mathcal{O}\left(-\sigma^{2} \log \sigma\right) \ll 1$ limit. In this way, we showed systematically how to replace the nonuniform boundary condition on the surface of the target sphere by an effective Robin boundary condition with a trapping rate $\kappa$ determined by the homogenized capacitance. The leading order term in $\kappa$ is the well-known Berg-Purcell result [4], and our analysis provides in a systematic way, with no fitting parameters, certain correction terms.

Finally, it would be interesting to determine the narrow capture time when one includes a more biologically realistic model of an individual nanotrap. One rather simplistic model for surface nanotraps is to assume that they can exhibit Markovian switching between an ON and OFF state, which roughly models the opening and closing of nanotraps on the cell nucleus. In [8] the related, but simpler, MFPT problem in which the entire boundary of a target sphere can switch between absorbing or reflecting was analyzed. However, a more biologically realistic class of surface nanotrap models is to consider a gated process in which a Brownian particle can switch states inside the boundary layer near the trap due to conformational changes of the ligand, as was discussed and modeled in [42]. Mathematically, with either class [42] or [8] of nanotrap models, one must extend our analysis of a scalar singularly perturbed elliptic problem to a two-component singularly perturbed elliptic system.

Appendix A. The exterior surface Green's function for a sphere. In this appendix we determine the local and far-field behavior of the surface Green's function for the unit sphere satisfying $(1.7 \mathrm{c})$ with a boundary singularity at $\theta_{0}, \phi_{0}$. The exact solution to (1.7) (cf. [35], [25]) is given in (1.7c). We first derive the local behavior (3.5) for $G$ as $\mathbf{y} \rightarrow \mathbf{y}_{0} \equiv\left(\sin \theta_{0} \cos \phi_{0}, \sin \theta_{0} \sin \phi_{0}, \cos \theta_{0}\right)^{T}$ in terms of the local variables

(A.1)

$\mathbf{z}=\sigma^{-1}\left(\mathbf{y}-\mathbf{y}_{0}\right), \quad \eta=\sigma^{-1}(r-1), \quad s_{1}=\sigma^{-1} \sin \theta_{0}\left(\phi-\phi_{0}\right), \quad s_{2}=\sigma^{-1}\left(\theta-\theta_{0}\right)$, 
where $r=|\mathbf{y}|$. To establish (3.5), we first use the law of cosines to get $\left|\mathbf{y}-\mathbf{y}_{0}\right|^{2}=$ $|\mathbf{y}|^{2}+1-2 \mathbf{y} \cdot \mathbf{y}_{0}$ since $\left|\mathbf{y}_{0}\right|=1$. This yields $\sigma^{2}|\mathbf{z}|^{2}=\left(1+2 \sigma \eta+\sigma^{2} \eta^{2}\right)+1-2 \mathbf{y} \cdot \mathbf{y}_{0}$, and so $1-\mathbf{y} \cdot \mathbf{y}_{0}=-\sigma \eta+\sigma^{2}\left(|\mathbf{z}|^{2}-\eta^{2}\right) / 2$. In this way, we calculate that

$$
1-\mathbf{y} \cdot \mathbf{y}_{0}+\left|\mathbf{y}-\mathbf{y}_{0}\right|=\sigma(|\mathbf{z}|-\eta)+\sigma^{2}\left(|\mathbf{z}|^{2}-\eta^{2}\right), \quad|\mathbf{y}|-\mathbf{y} \cdot \mathbf{y}_{0}=\frac{\sigma^{2}}{2}\left(|\mathbf{z}|^{2}-\eta^{2}\right) .
$$

Upon substituting (A.2) into (1.7c), and canceling a common factor of $|\mathbf{z}|-\sigma$, we obtain that

$\log \left(\frac{1-\mathbf{y} \cdot \mathbf{y}_{0}+\left|\mathbf{y}-\mathbf{y}_{0}\right|}{|\mathbf{y}|-\mathbf{y} \cdot \mathbf{y}_{0}}\right) \sim \log \left(\frac{2}{\sigma(|\mathbf{z}|+\eta)}+2\right) \sim-\log \left(\frac{\sigma}{2}\right)-\log (|\mathbf{z}|+\eta)+\mathcal{O}(\sigma)$.

Combining this relation with (1.7c), we obtain as $\mathbf{y} \rightarrow \mathbf{y}_{0}$ that

$$
G\left(\mathbf{y} ; \mathbf{y}_{0}\right) \sim \frac{1}{2 \pi \sigma|\mathbf{z}|}+\frac{1}{4 \pi} \log \left(\frac{\sigma}{2}\right)+\frac{1}{4 \pi} \log (|\mathbf{z}|+\eta)+\mathcal{O}(\sigma) .
$$

Next, we derive a two-term expansion for $|\mathbf{z}|^{-1}$ in terms of the local coordinates $\left(s_{1}, s_{2}, \rho\right)$, where $\rho \equiv \sqrt{s_{1}^{2}+s_{2}^{2}+\eta^{2}}$.

LEMmA A.1. In terms of the local coordinates in (A.1), we have that

$$
\frac{1}{|\mathbf{z}|}=\frac{1}{\rho}-\frac{\sigma}{2 \rho^{3}}\left[\eta\left(s_{1}^{2}+s_{2}^{2}\right)+s_{1}^{2} s_{2} \cot \theta_{k}\right]+\mathcal{O}\left(\sigma^{2}\right), \quad \text { where } \quad \rho \equiv \sqrt{\eta^{2}+s_{1}^{2}+s_{2}^{2}} .
$$

Proof. We write $\mathbf{y}=r(\sin \theta \cos \phi, \sin \phi \sin \theta, \cos \theta)^{T} \equiv\left(f_{1}(r, \phi, \theta), f_{2}(r, \phi, \theta)\right.$, $\left.f_{3}(r, \phi, \theta)\right)^{T}$. A Taylor series approximation to second order about $\mathbf{y}_{0}$ yields that $\mathbf{y}-\mathbf{y}_{0}=J \mathbf{h}+\frac{1}{2} \mathbf{e}+\cdots$, where $\mathbf{h} \equiv\left(r-1, \phi-\phi_{0}, \theta-\theta_{0}\right)^{T}$ and

$$
\begin{aligned}
J & \equiv=\left(\begin{array}{ccc}
\cos \phi_{0} \sin \theta_{0} & -\sin \phi_{0} \sin \theta_{0} & \cos \phi_{0} \cos \theta_{0} \\
\sin \phi_{0} \sin \theta_{0} & \cos \phi_{0} \sin \theta_{0} & \sin \phi_{0} \cos \theta_{0} \\
\cos \theta_{0} & 0 & -\sin \theta_{0}
\end{array}\right), \quad \mathbf{e} \equiv\left(\begin{array}{c}
\mathbf{h}^{T} H_{1} \mathbf{h} \\
\mathbf{h}^{T} H_{2} \mathbf{h} \\
\mathbf{h}^{T} H_{3} \mathbf{h}
\end{array}\right), \\
H_{j} & \equiv\left(\begin{array}{ccc}
f_{j r r}^{0} & f_{j r \phi}^{0} & f_{j r \theta}^{0} \\
f_{j r \phi}^{0} & f_{j \phi \phi}^{0} & f_{j \phi \theta}^{0} \\
f_{j r \theta}^{0} & f_{j \phi \theta}^{0} & f_{j \theta \theta}^{0}
\end{array}\right),
\end{aligned}
$$

where the $H_{j}$ are evaluated at $\mathbf{y}_{0}$. Setting $\mathbf{y}-\mathbf{y}_{0}=\sigma \mathbf{z}$, and writing $\mathbf{h}$ in terms of the local coordinates (A.1), we get

$$
|\mathbf{z}|=\left(\mathbf{h}_{0} J^{T} J \mathbf{h}_{0}+\sigma \mathbf{h}_{0}^{T} J^{T} \mathbf{e}_{0}+\mathcal{O}\left(\sigma^{2}\right)\right)^{1 / 2}, \quad \mathbf{h}_{0} \equiv\left(\begin{array}{c}
\eta \\
s_{1} / \sin \theta_{0} \\
s_{2}
\end{array}\right), \quad \mathbf{e}_{0} \equiv\left(\begin{array}{c}
\mathbf{h}_{0}^{T} H_{1} \mathbf{h}_{0} \\
\mathbf{h}_{0}^{T} H_{2} \mathbf{h}_{0} \\
\mathbf{h}_{0}^{T} H_{3} \mathbf{h}_{0}
\end{array}\right)
$$

We readily calculate that $\mathbf{h}_{0} J^{T} J \mathbf{h}_{0}=\rho^{2}=\left(\eta^{2}+s_{1}^{2}+s_{2}^{2}\right)$, so that by using the binomial theorem and by a straightforward but lengthy calculation of $J^{T} \mathbf{e}_{0}$, we obtain

$$
\frac{1}{|\mathbf{z}|}=\frac{1}{\rho}-\frac{\sigma}{2 \rho^{3}} \mathbf{h}_{0}^{T} J^{T} \mathbf{e}_{0}+\mathcal{O}\left(\sigma^{2}\right), \quad \text { where } \quad J^{T} \mathbf{e}_{0}=\left(\begin{array}{c}
-\left(s_{1}^{2}+s_{2}^{2}\right) \\
2 \eta s_{1} \sin \theta_{0}+2 s_{1} s_{2} \cos \theta_{0} \\
2 \eta s_{2}-s_{1}^{2} \cot \theta_{0}
\end{array}\right) .
$$

By calculating $\mathbf{h}_{0}^{T}\left(J^{T} \mathbf{e}_{0}\right)$ we get (A.4). Finally, upon substituting (A.4) into (A.3), we obtain the local behavior (3.5). 
Finally, we find the behavior of $G\left(\mathbf{y} ; \mathbf{y}_{0}\right)$ as $|\mathbf{y}| \rightarrow \infty$. From the law of cosines, $\left|\mathbf{y}-\mathbf{y}_{0}\right| \sim|\mathbf{y}|-\mathbf{y} \cdot \mathbf{y}_{0} /|\mathbf{y}|+\cdots$, so that

$$
\begin{aligned}
& \log \left(\frac{1-\mathbf{y} \cdot \mathbf{y}_{0}+\left|\mathbf{y}-\mathbf{y}_{0}\right|}{|\mathbf{y}|-\mathbf{y} \cdot \mathbf{y}_{0}}\right) \sim \log \left(\frac{1-\mathbf{y} \cdot \mathbf{y}_{0}+|\mathbf{y}|-\mathbf{y} \cdot \mathbf{y}_{0} /|\mathbf{y}|}{|\mathbf{y}|-\mathbf{y} \cdot \mathbf{y}_{0}}\right)=\log \left(1+\frac{1}{|\mathbf{y}|}\right) \sim \frac{1}{|\mathbf{y}|} \\
& \text { for }|\mathbf{y}| \gg 1 \text {. }
\end{aligned}
$$

From (1.7c) it follows that $G\left(\mathbf{y} ; \mathbf{y}_{0}\right) \sim[4 \pi|\mathbf{y}|]^{-1}$ as $|\mathbf{y}| \rightarrow \infty$. As a remark, if we carry the calculation to one higher order, we can obtain the dipole term in the far-field behavior explicitly as

$$
G\left(\mathbf{y} ; \mathbf{y}_{0}\right) \sim \frac{1}{4 \pi|\mathbf{y}|}+\frac{3}{8 \pi} \frac{\mathbf{y} \cdot \mathbf{y}_{0}}{|\mathbf{y}|^{3}}+\cdots \quad \text { as } \quad|\mathbf{y}| \rightarrow \infty .
$$

Appendix B. The inner problem for $\boldsymbol{w}_{2}$. In this appendix we summarize the analysis of the solution to (3.26) and (3.27), and the determination of the farfield behavior of $w_{2}$ in (3.25). The first result provides the explicit solution for $w_{2 e}$ satisfying (3.27).

Lemma B.1. The exact solution to (3.27) is

$$
w_{2 e}=\frac{\eta^{2}}{2} \partial_{\eta} w_{c}+\frac{\eta}{2} w_{c}-\frac{1}{2} \int_{0}^{\eta} w_{c}\left(z, s_{1}, s_{2}\right) d z-\mathcal{K}\left(s_{1}, s_{2}\right)+w_{2 h},
$$

where $w_{c}$ is the solution (3.11c) to the electrified disk problem (3.11). Here $\mathcal{K}\left(s_{1}, s_{2}\right)$ is the unique solution to the following Poisson's equation with a compactly supported inhomogeneous term:

$$
\begin{aligned}
& \Delta_{s} \mathcal{K} \equiv \mathcal{K}_{s_{1} s_{1}}+\mathcal{K}_{s_{2} s_{2}}=-\left.\frac{1}{2} \partial_{\eta} w_{c}\right|_{\eta=0} \\
& \mathcal{K}\left(s_{1}, s_{2}\right) \sim \frac{c_{k}}{2} \log |\mathbf{s}|+o(1) \quad \text { as } \quad|\mathbf{s}| \equiv\left(s_{1}^{2}+s_{2}^{2}\right)^{1 / 2} \rightarrow \infty
\end{aligned}
$$

where $c_{k}=2 a_{k} / \pi$. In terms of $\mathcal{K}\left(s_{1}, s_{2}\right)$, the function $w_{2 h}$ in (B.1) satisfies the half-space problem formulated as

$$
\mathcal{L} w_{2 h}=0, \quad \eta>0, \quad-\infty<s_{1}, s_{2}<\infty,
$$

$$
w_{2 h}=\mathcal{K}\left(s_{1}, s_{2}\right), \quad \eta=0, \quad s_{1}^{2}+s_{2}^{2}<a_{k}^{2} ; \quad \partial_{\eta} w_{2 h}=0, \quad \eta=0, \quad s_{1}^{2}+s_{2}^{2} \geq a_{k}^{2},
$$

$$
w_{2 h}=\mathcal{O}\left(\rho^{-1}\right) \quad \text { as } \quad \rho=\left(\eta^{2}+s_{1}^{2}+s_{2}^{2}\right)^{1 / 2} \rightarrow \infty,
$$

where $\mathcal{L} \equiv \partial_{\eta \eta}+\partial_{s_{1} s_{1}}+\partial_{s_{2} s_{2}}$. The far-field behavior for $w_{2 h}$ is

$$
w_{2 h} \sim \frac{c_{k} b_{k}}{\rho} \quad \text { as } \rho \rightarrow \infty, \quad \text { where } b_{k} \equiv \frac{c_{k}}{2}\left(\log \left(4 a_{k}\right)-\frac{3}{2}\right) .
$$

The solution $w_{2 e}$ to (3.27), written in (B.1), has the far-field behavior

$$
w_{2 e} \sim-\frac{c_{k}}{2} \log (\eta+\rho)+\frac{c_{k}}{2 \rho^{3}} \eta\left(s_{1}^{2}+s_{2}^{2}\right)+\frac{c_{k} b_{k}}{\rho}+\cdots \quad \text { as } \quad \rho \rightarrow \infty .
$$


Before proving this result, we remark that the $o(1)$ condition in the far-field behavior (B.2) and the decay condition in (B.3c) determine $\mathcal{K}\left(s_{1}, s_{2}\right)$ and $w_{2 h}$ uniquely. In addition, upon using the divergence theorem on the problem (3.11) for $w_{c}$, it readily follows that the far-field behavior in (B.2) for $\mathcal{K}$ has the correct strength for the logarithmic singularity.

Proof. We must first establish that (B.1) accounts for the inhomogeneous term in the PDE (3.27). Then we must show that both the boundary conditions (3.27b) and the required far-field behavior (3.27c) are satisfied. We first verify that (B.1) satisfies the PDE (3.27). We write $\mathcal{L} v \equiv v_{\eta \eta}+\Delta_{s} v$, where $\Delta_{s} v \equiv v_{s_{1} s_{1}}+v_{s_{2} s_{2}}$, and calculate that

$\mathcal{L} w_{2 e}=\frac{\eta^{2}}{2} \partial_{\eta \eta \eta} w_{c}+\frac{5}{2} \eta \partial_{\eta \eta} w_{c}+\frac{3}{2} \partial_{\eta} w_{c}+\frac{\eta^{2}}{2} \partial_{\eta}\left(\Delta_{s} w_{c}\right)+\frac{\eta}{2} \Delta_{s} w_{c}-\frac{1}{2} \int_{0}^{\eta} \Delta_{s} w_{c} d z-\Delta_{s} \mathcal{K}$.

Then, by using the PDE $\partial_{\eta \eta} w_{c}=-\Delta_{s} w_{c}$ satisfied by $w_{c}$, the expression above becomes

$$
\begin{aligned}
\mathcal{L} w_{2 e}= & \frac{\eta^{2}}{2} \partial_{\eta \eta \eta} w_{c}+\frac{5}{2} \eta \partial_{\eta \eta} w_{c}+\frac{3}{2} \partial_{\eta} w_{c}-\frac{\eta^{2}}{2} \partial_{\eta \eta \eta} w_{c}-\frac{\eta}{2} \partial_{\eta \eta} w_{c} \\
& +\frac{1}{2}\left(\partial_{\eta} w_{c}-\left.\partial_{\eta} w_{c}\right|_{\eta=0}\right)-\Delta_{s} \mathcal{K}=2\left(\eta \partial_{\eta \eta} w_{c}+\partial_{\eta} w_{c}\right)
\end{aligned}
$$

as desired, provided that $\Delta_{s} \mathcal{K}=-\left.\frac{1}{2} \partial_{\eta} w_{c}\right|_{\eta=0}$. Therefore, $w_{2 e}$ satisfies the PDE (3.27a) when $\mathcal{K}$ satisfies (B.2).

Next, we observe from (B.1) that on $\eta=0$ the boundary conditions $\partial_{\eta} w_{2 e}=0$ for $s_{1}^{2}+s_{2}^{2}>a_{k}^{2}$ and $w_{2 e}=0$ for $s_{1}^{2}+s_{2}^{2}<a_{k}^{2}$ are satisfied, provided that $w_{2 h}$ satisfies the boundary conditions in (B.3b).

To establish the far-field behavior (B.3d) for $w_{2 h}$ we analyze the problem (B.3) for $w_{2 h}$. By using (3.11c), we first calculate $\left.\partial_{\eta} w_{c}\right|_{\eta=0}$ on the nanotrap. Upon defining $|\mathbf{s}| \equiv \sqrt{s_{1}^{2}+s_{2}^{2}}$, we conclude from (B.2) that $\mathcal{K}$ satisfies

$$
\Delta_{s} \mathcal{K}= \begin{cases}\pi^{-1}\left[a_{k}^{2}-|\mathbf{s}|^{2}\right]^{-1 / 2}, & 0 \leq|\mathbf{s}|<a_{k} \\ 0, & |\mathbf{s}| \geq a_{k}\end{cases}
$$

The unique solution to (B.5) that satisfies the far-field condition in (B.2) depends only on $|\mathbf{s}|$ and is

$$
\mathcal{K}(|\mathbf{s}|)= \begin{cases}\frac{1}{\pi}\left[a_{k} \log \left(a_{k}+\sqrt{a_{k}^{2}-|\mathbf{s}|^{2}}\right)-\sqrt{a_{k}^{2}-|\mathbf{s}|^{2}}\right], & 0 \leq|\mathbf{s}| \leq a_{k} \\ \frac{a_{k}}{\pi} \log |\mathbf{s}|, & |\mathbf{s}| \geq a_{k}\end{cases}
$$

Then, by using this explicit expression for $K(|\mathbf{s}|)$ for $0<|\mathbf{s}|<a_{k}$ in the boundary condition for $w_{2 h}$ in (B.3) on the nanotrap, we can calculate the monopole behavior of $w_{2 h}$ as $\rho \rightarrow \infty$ in (B.3d) by using the known far-field behavior

$$
w_{2 h} \sim \frac{2}{\pi \rho} \int_{0}^{a_{k}} \frac{\mathcal{K}(|\mathbf{s}|)|\mathbf{s}|}{\sqrt{a_{k}^{2}-|\mathbf{s}|^{2}}} d|\mathbf{s}| \quad \text { as } \quad \rho \rightarrow \infty,
$$

as given in [18] and section 1.4 of [19]. The integral in (B.7) is readily evaluated to yield the far-field behavior in (B.3d).

Finally, with the far-field behavior of $w_{2 h}$ now known, we next determine the asymptotic far-field behavior of $w_{2 e}$ as defined in (B.1). We use $w_{c} \sim c_{j} \rho^{-1}$ as 
$\rho \rightarrow \infty$ with $\rho=\left(\eta^{2}+|\mathbf{s}|^{2}\right)^{1 / 2}$ and $|\mathbf{s}|=\left(s_{1}^{2}+s_{2}^{2}\right)^{1 / 2}$ to calculate

$$
\frac{1}{2} \eta^{2} \partial_{\eta} w_{c}+\frac{1}{2} \eta w_{c} \sim \frac{c_{k}}{2} \frac{\eta|\mathbf{s}|^{2}}{\left(\eta^{2}+|\mathbf{s}|^{2}\right)^{3 / 2}} \quad \text { as } \quad \rho \rightarrow \infty,
$$

$\frac{1}{2} \int_{0}^{\eta} w_{c}\left(z, s_{1}, s_{2}\right) d z \sim \frac{c_{k}}{2} \int_{0}^{\eta} \frac{1}{\left(z^{2}+|\mathbf{s}|^{2}\right)^{1 / 2}} d z=\frac{c_{k}}{2}\left[\log \left(\eta+\sqrt{\eta^{2}+|\mathbf{s}|^{2}}\right)-\log |\mathbf{s}|\right]$

$$
\text { as } \rho \rightarrow \infty \text {. }
$$

Since $\mathcal{K} \sim \frac{c_{k}}{2} \log |\mathbf{s}|+o(1)$ as $|\mathbf{s}| \rightarrow \infty$ cancels the last term in (B.8b), $w_{2 e}$ in (B.1) has the far-field behavior (B.4).

Lemma B.2. The exact solution to (3.26) is given explicitly by

$$
w_{2 o}=\cot \theta_{k}\left(\frac{s_{1}^{2}}{2} \partial_{s_{2}} w_{c}-s_{2} s_{1} \partial_{s_{1}} w_{c}\right)
$$

Proof. We first verify that $w_{2 o}$ satisfies the PDE (3.26). Upon using $\mathcal{L}\left(\partial_{s_{1}} w_{c}\right)=0$ and $\mathcal{L}\left(\partial_{s_{2}} w_{c}\right)=0$, we calculate

$$
\begin{aligned}
\mathcal{L} w_{2 o} & =\cot \theta_{k}\left[\frac{1}{2} \mathcal{L}\left(s_{1}^{2} \partial_{s_{2}} w_{c}\right)-\mathcal{L}\left(s_{1} s_{2} \partial_{s_{1}} w_{c}\right)\right] \\
& =\cot \theta_{k} v\left[\frac{1}{2}\left(4 s_{1} \partial_{s_{2} s_{1}} w_{c}+2 \partial_{s_{2}} w_{c}\right)-\left(2 s_{2} \partial_{s_{1} s_{1}} w_{c}+2 s_{1} \partial_{s_{2} s_{1}} w_{c}\right)\right] \\
& =\cot \theta_{k}\left(\partial_{s_{2}} w_{c}-2 s_{2} \partial_{s_{1} s_{1}} w_{c}\right)
\end{aligned}
$$

as required. Next, we verify the boundary conditions in (3.26b). Since $w_{c}=1$ on the nanotrap, it follows that $\partial_{s_{1}} w_{c}=0, \partial_{s_{2}} w_{c}=0$, and consequently $w_{2 o}=0$, on the nanotrap. Off the nanotrap, we have $\partial_{\eta} w_{c}=0$, so that by differentiating (B.9) in $\eta$ it follows that $\partial_{\eta} w_{2 o}=0$ holds off the nanotrap. Finally, we use $w_{c} \sim c_{k} / \rho$ as $\rho \rightarrow \infty$ to find that the far-field condition (3.26c) holds exactly, and consequently there is no monopole term in the far-field behavior of $w_{2 o}$.

Appendix C. Distribution of points on a sphere. The equidistribution of a fixed number of points on the surface of a sphere is a long-studied problem in approximation theory [44] and arises in many disparate fields. An easy-to-implement algorithm which produces a very homogeneously distributed set of points is the Fibonacci lattice $[21,49]$. Starting from an integer $M$, this algorithm produces $N=2 M+1$ points on the sphere with the $k$ th point given in spherical coordinates by

$$
\sin \theta_{k}=\frac{2 k}{N}, \quad \phi_{k}=\frac{2 \pi k}{\Phi},
$$

where $\Phi=1+\Phi^{-1}=(1+\sqrt{5}) / 2 \approx 1.618$ is the golden ratio. This can be implemented in a few lines of MATLAB code:

$\%$ Fibonacci Lattice Points

$\mathrm{M}=400 ; \quad \mathrm{N}=2 * \mathrm{M}+1$;

theta $=\mathrm{pi} / 2-\operatorname{asin}(2 *(-\mathrm{M}: \mathrm{M}) / \mathrm{N})$;

phi $=4 *$ pi* $(-\mathrm{M}: \mathrm{M}) /(1+\operatorname{sqrt}(5))$;

$\mathrm{x}=\sin ($ theta) $\cdot * \cos (\mathrm{phi}) ; \quad \mathrm{y}=\sin ($ theta $) \cdot * \sin (\mathrm{phi}) ; \quad \mathrm{z}=\cos ($ theta $) ;$ 


\section{REFERENCES}

[1] O. BÉnichou And R. Voituriez, From first-passage times of random walks in confinement to geometry-controlled kinetics, Phys. Rep., 539 (2014), pp. 225-284.

[2] A. M. Berezhrovskit, Y. A. Makhnovskit, M. I. Monine, V. Y. Zitserman, and S. Y. ShVARTSMan, Boundary homogenization for trapping by patchy surfaces, J. Chem. Phys., 121 (2004), pp. 11390-11394.

[3] A. M. Berezhrovskit, M. I. Monine, C. B. Muratov, and S. Y. Shvartsman, Homogenization of boundary conditions for surfaces with regular arrays of traps, J. Chem. Phys., 124 (2006), 036103

[4] H. C. Berg and E. M. Purcell, Physics of chemoreception, Biophys. J, 20 (1977), pp. 193219.

[5] B. Bergersen, D. Boal, And P. Palffy-Muhoray, Equilibrium configurations of particles on the sphere: The case of logarithmic interactions, J. Phys. A Math Gen., 27 (1994), pp. 2579-2586.

[6] A. Bernoff And A. Lindsay, Numerical approximation of diffusive capture rates by planar and spherical surfaces with absorbing pores, to be submitted.

[7] L. Bétermin And E. SAndier, Renormalized Energy and Asymptotic Expansion of Optimal Logarithmic Energy on the Sphere, preprint, arXiv:1404.4485 [math.AP], 2015.

[8] P. Bressloff and S. D. Lawley, Stochastically gated diffusion-limited reactions for a small target in a bounded domain, Phys. Rev. E, 92 (2015), 062117.

[9] P. C. Bressloff And J. M. Newby, Stochastic models of intracellular transport, Rev. Modern Phys., 85 (2013), pp. 135-196, https://doi.org/10.1103/RevModPhys.85.135.

[10] X. Chen And A. Friedman, Asymptotic analysis for the narrow escape problem, SIAM J. Math. Anal., 43 (2011), pp. 2542-2563, https://doi.org/10.1137/090775257.

[11] A. Cheviakov and M. J. Ward, Optimizing the principal eigenvalue of the Laplacian in a sphere with interior traps, Math. Comput. Modelling, 53 (2011), pp. 1394-1409.

[12] A. F. Cheviakov, M. J. Ward, and R. Straube, An asymptotic analysis of the mean first passage time for narrow escape problems: Part II: The sphere, Multiscale Model. Simul., 8 (2010), pp. 836-870, https://doi.org/10.1137/100782620.

[13] A. Cheviakov and D. Zawada, Narrow-escape problem for the unit sphere: Homogenization limit, optimal arrangements of large number of traps, and the $n^{2}$ conjecture, Phys. Rev. E, 87 (2013), 042118.

[14] W. D. Collins, On some dual series equations and their application to electrostatic problems for spheroidal caps, Math. Proc. Cambridge Phil. Soc., 57 (1961), pp. 367-384.

[15] D. Coombs, R. Straube, And M. Ward, Diffusion on a sphere with localized traps: Mean first passage time, eigenvalue asymptotics, and Fekete points, SIAM J. Appl. Math., 70 (2009), pp. 302-332, https://doi.org/10.1137/080733280.

[16] M. I. Delgado, M. J. Ward, And D. Coombs, Conditional mean first passage times to small traps in a 3-D domain with a sticky boundary: Applications to $T$ cell searching behavior in lymph nodes, Multiscale Model. Simul., 13 (2015), pp. 1224-1258, https://doi.org/10. $1137 / 140978314$.

[17] D. G. Duffy, Mixed Boundary Value Problems, Chapman and Hall/CRC, Boca Raton, FL, 2008.

[18] A. B. Efimov and V. N. Vorobev, A mixed-boundary value problem for the Laplace equation, J. Engrg. Phys., 26 (1974), pp. 664-666.

[19] V. I. Fabrikant, ED., Applications of Potential Theory to Mechanics: A Selection of New Results, Kluwer Academic, 1989.

[20] E. Glasser And A. G. Every, Energies and spacings of point charges on the sphere, J. Phys. A Math. Gen., 25 (1992), pp. 2473-2482.

[21] Á. GonzÁlez, Measurement of areas on a sphere using Fibonacci and latitude-longitude lattices, Math. Geosci., 42 (2009), pp. 49-64, https://doi.org/10.1007/s11004-009-9257-x.

[22] D. Holcman and T. Lagache, Narrow Escape to Small Windows on a Small Ball Modeling the Viral Entry into the Cell Nucleus, preprint, arXiv:1607.02479 [q-bio.SC], 2016.

[23] D. Holcman and Z. Schuss, The narrow escape problem, SIAM Rev., 56 (2014), pp. 213-257, https://doi.org/10.1137/120898395.

[24] S. A. IsAaCSON AND J. M. NewBy, Uniform asymptotic approximation of diffusion to a small target, Phys. Rev. E, 88 (2013), 012820, https://doi.org/10.1103/PhysRevE.88.012820.

[25] O. D. Kellog, Foundations of Potential Theory, Dover, New York, 1954.

[26] T. Kolokolnikov, M. S. Titcombe, and M. J. Ward, Optimizing the fundamental Neumann eigenvalue for the Laplacian in a domain with small traps, European J. Appl. Math., 16 (2005), pp. 161-200, https://doi.org/10.1017/S0956792505006145. 
[27] A. B. J. Kuijlaars and E. B. SafF, Asymptotics for minimal discrete energy on the sphere, Trans. Amer. Math. Soc., 350 (1998), pp. 523-538.

[28] T. Lagache, E. Dauty, and D. Holcman, Quantitative analysis of virus and plasmid trafficking in cells, Phys. Rev. E, 79 (2009), 011921.

[29] T. Lagache and D. Holcman, Quantifying intermittent transport in cell cytoplasm, Phys. Rev. E, 77 (2008), 030901.

[30] P. A. Lagerstrom and D. A. Reinelt, Note on logarithmic switchback terms in regular and singular perturbation problems, SIAM J. Appl. Math., 44 (1984), pp. 451-462, https://doi. org/10.1137/0144030.

[31] D. A. Lauffenburger and J. J. Linderman, Receptors: Models for Binding, Trafficking and Signalling, Oxford University Press, New York, 1993.

[32] A. E. Lindsay, T. Kolokolnikov, and J. C. Tzou, Narrow escape problem with a mixed trap and the effect of orientation, Phys. Rev. E, 91 (2015), 032111, https://doi.org/10.1103/ PhysRevE.91.032111.

[33] A. E. Lindsay, R. Spoonmore, and J. Tzou, Hybrid asymptotic-numerical approach for estimating first-passage-time densities of the two-dimensional narrow capture problem, Phys. Rev. E, 94 (2016), 042418.

[34] C. B. Muratov and S. Y. Shvartsman, Boundary homogenization for periodic arrays of absorbers, Multiscale Model. Simul., 7 (2008), pp. 44-61, https://doi.org/10.1137/070692832.

[35] I. M. Nemenman AND A. S. Silbergleit, Explicit Green's function of a boundary value problem for a sphere and trapped flux analysis in Gravity Probe B experiment, J. Appl. Phys., 86 (1999), pp. 614-624.

[36] P. Novák AND J. NovÁK, Efficient and stable numerical method for evaluation of Zernike polynomials and their Cartesian derivatives, Proc. SPIE, 8789 (2013), 878913, https:// doi.org/10.1117/12.2020389.

[37] S. Pillay, M. J. Ward, A. Peirce, and T. Kolokolnikov, An asymptotic analysis of the mean first passage time for narrow escape problems: Part I: Two-dimensional domains, Multiscale Model. Simul., 8 (2010), pp. 803-835, https://doi.org/10.1137/090752511.

[38] M. R., O. G., And S. Redner, EdS., First-Passage Phenomena and Their Applications, World Scientific, 2014.

[39] E. A. Rakhamanov, E. B. Saff, and Y. M. Zhou, Minimal discrete energy on the sphere, Math. Res. Lett., 1 (1994), pp. 647-662.

[40] S. Redner, A Guide to First-Passage Processes, Cambridge University Press, Cambridge, UK, 2001.

[41] J. Reingruber, E. Abad, And D. Holcman, Narrow escape time to a structured target located on the boundary of a microdomain, J. Chem. Phys., 130 (2009), 094909.

[42] J. Reingruber and D. Holcman, Gated narrow escape time for molecular signaling, Phys. Rev. Lett., 103 (2009), 148102.

[43] J. F. Rupprecht, O. Bénichou, D. S. Grebenkov, and R. Voituriez, Exit time distribution in spherically symmetric two-dimensional domains, J. Stat. Phys., 158 (2015), pp. 192-230.

[44] E. B. Saff And A. B. J. KuiJlaArs, Distributing many points on a sphere, Math. Intelligencer, 19 (1997), pp. 5-11.

[45] D. Shoup AND A. SzABo, Role of diffusion in ligand binding to macromolecules and cell-bound receptors, Biophys. J, 40 (1982), pp. 33-39.

[46] A. Singer, Z. Schuss, and D. Holcman, Narrow escape, Part II: The circular disk, J. Stat. Phys., 122 (2006), pp. 465-489, https://doi.org/10.1007/s10955-005-8027-5.

[47] A. Singer, Z. Schuss, D. Holcman, And R. S. Eisenberg, Narrow escape, Part I, J. Stat. Phys., 122 (2006), pp. 437-463.

[48] I. N. Sneddon, Mixed Boundary Value Problems in Potential Theory, North-Holland, 1966.

[49] R. Swinbank and R. J. Purser, Fibonacci grids: A novel approach to global modelling, Quart. J. Roy. Meteorolog. Soc., 132 (2006), pp. 1769-1793, https://doi.org/10.1256/qj.05.227.

[50] M. Webster, K. L. Witkin, And O. Cohen-Fix, Sizing up the nucleus: Nuclear shape, size and nuclear-envelope assembly, J. Cell Sci., 122 (2009), pp. 1477-1486, https://doi.org/ 10.1242/jcs.037333, https://arxiv.org/abs/http://jcs.biologists.org/content/122/10/1477. full.pdf.

[51] R. ZwanZig, Diffusion controlled ligand binding to spheres partially covered by receptors: An effective medium treatment, Proc. Natl. Acad. Sci. USA, 87 (1990), pp. 5856-5857. 